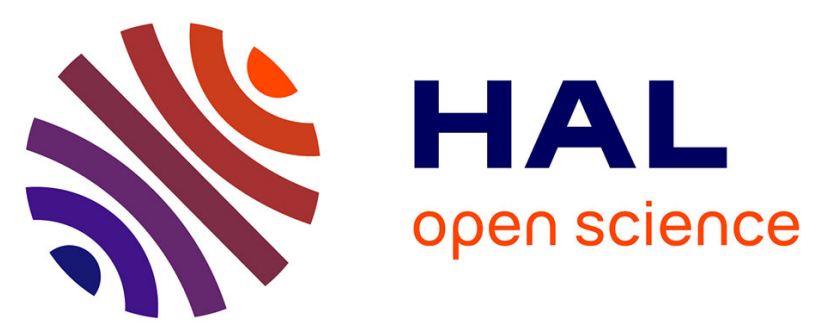

\title{
Discontinuous Galerkin finite element method applied to the coupled unsteady Stokes/Cahn-Hilliard equations
} Franck Pigeonneau, Elie Hachem, Pierre Saramito

\section{To cite this version:}

Franck Pigeonneau, Elie Hachem, Pierre Saramito. Discontinuous Galerkin finite element method applied to the coupled unsteady Stokes/Cahn-Hilliard equations. International Journal for Numerical Methods in Fluids, 2019, 90 (6), pp.267-295. 10.1002/fld.4720 . hal-01773404v2

HAL Id: hal-01773404

https://hal-mines-paristech.archives-ouvertes.fr/hal-01773404v2

Submitted on 14 Feb 2019

HAL is a multi-disciplinary open access archive for the deposit and dissemination of scientific research documents, whether they are published or not. The documents may come from teaching and research institutions in France or abroad, or from public or private research centers.
L'archive ouverte pluridisciplinaire HAL, est destinée au dépôt et à la diffusion de documents scientifiques de niveau recherche, publiés ou non, émanant des établissements d'enseignement et de recherche français ou étrangers, des laboratoires publics ou privés. 
DOI: $\mathrm{xxx} / \mathrm{xxxx}$ 10.1002/fld.4720

\title{
RESEARCH ARTICLE
}

\section{Discontinuous Galerkin finite element method applied to the coupled unsteady Stokes/Cahn-Hilliard equations}

\author{
F. Pigeonneau*1 | E. Hachem ${ }^{1}$ | P. Saramito ${ }^{2}$
}

\author{
${ }^{1}$ MINES ParisTech, PSL Research \\ University, CEMEF - Centre for material \\ forming CNRS UMR 7635, CS 10207, \\ Claude Daunesse 06904 Sophia Antipolis \\ cedex, France \\ ${ }^{2}$ CNRS, Lab. J. Kuntzmann, B.P. 53, 38041 \\ Grenoble cedex 9, France \\ Correspondence \\ *F. Pigeonneau, MINES ParisTech, PSL \\ Research University, CEMEF - Centre for \\ material forming, CNRS UMR 7635. Email: \\ franck.pigeonneau@mines-paristech.fr
}

\begin{abstract}
Two-phase flows driven by the interfacial dynamics are studied by tracking implicitly interfaces in the framework of the Cahn-Hilliard theory. The fluid dynamics is described by the Stokes equations with an additional source term in the momentum equation taking into account the capillary forces. A discontinuous Galerkin finite element method is used to solve the coupled Stokes/Cahn-Hilliard equations. The Cahn-Hilliard equation is treated as a system of two coupled equations corresponding to the advection-diffusion equation for the phase field and a non-linear elliptic equation for the chemical potential. First, the variational formulation of the Cahn-Hilliard equation is presented. A numerical test is achieved showing the optimal-order in error bounds. Second, the variational formulation in discontinuous Galerkin finite element approach of the Stokes equations is recalled in which the same space of approximation is used for the velocity and the pressure with an adequate stabilization technique. The rates of convergence in space and time are evaluated leading to an optimal-order in error bounds in space and a second order in time with a backward differentiation formula at the second order. Numerical tests devoted to two-phase flows are provided on ellipsoidal droplet retraction, on the capillary rising of a liquid in a tube and on the wetting drop over a horizontal solid wall.

\section{KEYWORDS:}

two-phase flow, phase-field theory, finite element, discontinuous Galerkin, droplet retraction, capillary rising
\end{abstract}

\section{1 | INTRODUCTION}

Two-phase flows occur in many industrial processes as in metallurgy, nuclear plants, polymer and glass processing but also in nature such as volcano eruptions, ocean dynamics. An accurate description of the interfaces between immiscible fluids becomes important in particular when we need to study the wetting or dewetting on a substrate which can be chemically heterogeneous or structured at microscale or nanoscale. Therefore, the numerical simulation of such industrial processes needs to gain in accuracy.

Indeed, in the literature, many authors work on various numerical methods to simulate the dynamics of two-phase flows driven by the capillary forces. In ${ }^{1,2}$, the authors propose to track explicitly the interfaces using boundary integral method in the limit of vanishing Reynolds number whereas Unverdi and Tryggvason ${ }^{3}$ used a front tracking method when the Reynolds number is finite. These approaches are based on the Lagrangian description of interfaces. Another method tracking interfaces but also meshes in bulk is the Arbitrary Lagrangian Eulerian (ALE) technique initially proposed by Hirt et al. ${ }^{4}$. Nowadays, the ALE 
method is used in various cases as developed in references ${ }^{5,6,7}$. Despite the high level of accuracy of these techniques, both the topological changes and the contact line dynamics remain very challenging to investigate accurately.

Another set of numerical methods are designed to track the interfaces implicitly using a volume marker. They are known as the "Volume of Fluid" technique initially developed by Hirt and Nichols ${ }^{8}$ or as the "level-set" method proposed by Sethian ${ }^{9}$ and Sethian and Smereka ${ }^{10}$. They are based on the Eulerian description and can straightforward describe topological changes like coalescence or break-up of bubbles or drops. More recently, the behavior of the triple line has been implemented and validated using both approaches as shown in ${ }^{11,12}$. Note that Gross and Reusken ${ }^{13}$ summarized the various implicit tracking methods very clearly and accurately.

In addition of these two main approaches of interface tracking, new numerical methods have been recently developed to combine the level-set method and the interface tracking by Enright et al. ${ }^{14}$ and more recently by Bui et al. ${ }^{15}$. In other direction, Noble et al. ${ }^{16}$ developed a conformal decomposition finite element method in which nodes are added in the interfaces. This method has been recently extended by Fries ${ }^{17}$ with a higher-order finite element method.

Recall that, in both approaches, the interfaces are considered sharp which naturally lead to a singular behavior at the triple line, as explained in the Huh-Scriven's paradox ${ }^{18}$. Therefore, to regularize the physics at the triple line, a slip length is commonly introduced ${ }^{19}$. To overcome such singularities, new alternative methods consider the interfaces not sharp, i.e. with a small thickness. These techniques employ what we know as the concept of "phase-field" introduced initially in statistical physics to describe phase change or spinodal decomposition ${ }^{20}$. The development of the phase-field method in fluid mechanics has been summarized by Anderson et al. ${ }^{21}$. One of the first contribution of the application of the phase-field theory to two-phase flows has been proposed by Antanovskii ${ }^{22}$. It is worth mentioning that the main advantage of the phase-field method is that the normal and the curvature are not required to determine the capillary forces involved in the momentum equation. For sharp interfaces, the topological changes like coalescence between two fluid inclusions are purely geometry while with a phase-field model the short range interactions as van der Waals forces are implicitly taken into account ${ }^{23}$. Lowengrub and Truskinovsky ${ }^{24}$ developed a Cahn-Hilliard model and proved how the diffuse model converges toward a sharp interface limit. The diffuse interface model is also used to study the multiphase flows in microfluidics ${ }^{25}$.

This technique is also particularly well-adapted to study interaction with a solid substrate. The contact-line dynamics has been studied by Seppecher ${ }^{26}$ who showed that the removal of the singularity at the triple line is due to a mass transfer across the interface combined with a finite thickness of the interface. Jacqmin ${ }^{27}$ studied the dynamics of contact-line using the diffuse interface investigating the behaviors of the phase-field and the chemical potential close to the triple line. A variational formulation has been proposed by Qian et al. ${ }^{28}$ mainly to generalize the Navier boundary condition. The capillary-driven flows have been also studied by Villanueva and Amberg ${ }^{29}$. They investigated the wetting of drop on a solid substrate and the imbibition of a liquid in a porous media. Biben and Joly ${ }^{30}$ provided a contribution to study the wetting on nanostructured surfaces. They used a bottom-up approach starting from a lattice Boltzmann model to go forward a Cahn-Hilliard equation taking into account a surface wall energy. Yue et al. ${ }^{31}$ and Yue and Feng ${ }^{32}$ studied the effect of the diffuse thickness to represent adequately the sharp-interface limit.

However, the numerical solution of the non-linear and biharmonic Cahn-Hilliard equation coupled to fluid dynamics is not straightforward, in particular when dealing with complex geometries. Indeed, the typical size over which the phase field changes across the interface is small and thus requires a high spatial resolution. Moreover, far away from the interface, the diffusion of the chemical potential is weak meaning that the Cahn-Hilliard equation is a quasi-pure transport equation. Jacqmin ${ }^{33}$ implemented a compact-finite difference scheme to solve the Cahn-Hilliard/Navier-Stokes equations. Badalassi et al. ${ }^{34}$ developed a timesplitting method with a spectral method in space. Based on a finite element method with a mesh adaptation, Villanueva and Amberg ${ }^{29}$ developed a symbolic tool to solve the Cahn-Hilliard/Navier-Stokes equations.

In the present work, a discontinuous Galerkin finite element method has been chosen. The main feature of the technique is its mass conservation accuracy applied to convective equation ${ }^{35}$. Di Pietro and $\operatorname{Ern}^{36}$ provided the basic concept of a discontinuous Galerkin finite element technique in the goal to design and analyze this method in various cases. Error analysis for the Stokes problem in the framework of discontinuous Galerkin method has been studied under minimal regularity by Badia et al. ${ }^{37}$. Recall that the numerical computation using a discontinuous Galerkin method to solve the phase-field equation has been initiated by Feng and Karakashian ${ }^{38}$ among others. According to a method proposed by Baker ${ }^{39}$ who developed a discontinuous Galerkin method for biharmonic equation, Feng and Karakashian ${ }^{38}$ established an optimal-order error bound by solving the fourth-order equation when the polynomial degree is greater or equal to three. Kay et al. ${ }^{40}$ solved the Cahn-Hilliard equation as a system of two second-order equations. This method is close to the technique to solve biharmonic equations splitting in two elliptic equations. Gudi et al. ${ }^{41}$ employed this method with a discontinuous Galerkin method and proved the existence and uniqueness of the discrete 
problem. A discontinuous Galerkin formulation has been also developed by Wells et al. ${ }^{42}$ in which the formulation is done on the fourth-order equation and on the mixed finite element formulation in which the Cahn-Hilliard equation is decomposed in two second-order equations. Numerical analysis of Cahn-Hilliard-Navier-Stokes equations have been investigated by Liu and Rivière $^{43}$. These authors proved the solvability both for symmetric and non-symmetric penalties of the discontinuous Galerkin formulation. Nevertheless, this work is performed for uniform viscosity and density.

In our contribution, we extend the phase-field method to study the fluid dynamics but at small scale, thus the fluid inertia will be neglected. Consequently, we investigate the coupled Stokes/Cahn-Hilliard equations with a discontinuous Galerkin finite element technique. Due to the viscosity variation over the interfaces, the generalization of the Stokes formulation is provided for heterogeneous viscosity. We focus our investigation on the accuracy of the numerical solver and on the interaction between the two fluid phases and a substrate. A particular attention will be also devoted to the mass conservation.

The problem statement is presented in section 2 in which the Stokes/Cahn-Hilliard equations will be detailed. The numerical method solving the phase-field equation is presented in section 3 with a test to determine the truncation errors. The mass conservation between phases is also investigated. Section 4 is devoted to the numerical method for the coupled Stokes/CahnHilliard equations. The space and time rates of convergence will be also studied. Finally, this section will be concluded with the implementation of a mesh adaptation procedure. Two-phase flows will be investigated in section 5 with a first example describing the droplet retraction and a second case devoted to a capillary rising in which the flow is driven by the contact line dynamics. The last problem is devoted to a drop wetting on a horizontal substrate.

\section{2 | PHASE-FIELD FORMULATION OF TWO-PHASE FLOWS MODELING}

Two phases are considered with respectively a density $\rho_{1}$ and a dynamic viscosity $\eta_{1}$ for the phase 1 and $\rho_{2}$ and $\eta_{2}$ for the phase 2. The phase field method considers that the shift between the two phases occurs over a thin layer equal to $\zeta$ corresponding to the "diffuse interface" thickness. Conventionally, the phase 1 is taken as the heavy phase, i.e. $\rho_{1}>\rho_{2}$. In a space domain, $\Omega \subset \mathbb{R}^{d}$ with its boundary $\partial \Omega$ and $d=2$ or 3, the phase at any material point with a position $x$ and at each time $t$ is described by an "order parameter" $\varphi(x, t)$. By convention, the phase 1 is given by the order parameter $\varphi=1$ and the phase 2 by $\varphi=-1$. The function $\varphi$ can be also seen as a volume fraction meaning that the density written at every point of $\Omega$ is given by

$$
\rho(\varphi)=\frac{\rho_{1}+\rho_{2}}{2}+\frac{\rho_{1}-\rho_{2}}{2} \varphi .
$$

The dynamic viscosity is generally given as a function of $\varphi$ as follows

$$
\eta(\varphi)=\frac{\eta_{1}+\eta_{2}}{2}+\frac{\left(\eta_{1}-\eta_{2}\right)}{2} \varphi
$$

Under the actions of external forces, boundary conditions and interaction between the two phases, the media changes in space and time requiring the balance equations of mass and momentum. Moreover, the dynamics between the two phases has to be considered by writing an equation on the phase field $\varphi$.

Let start by the description from the thermodynamic equilibrium point of view. The total free energy of the system writes as a functional of $\varphi$ and its gradient as follows ${ }^{27,31}$

$$
\mathcal{F}[\varphi]=\int_{\Omega} f_{m}(\varphi, \nabla \varphi) d V+\int_{\partial \Omega_{w}} f_{w}(\varphi) d S,
$$

Functions $f_{m}(\varphi, \boldsymbol{\nabla} \varphi)$ and $f_{w}(\varphi)$ are respectively the mixing free energy in volume and the wall free energy ${ }^{44,27}$. The boundary $\partial \Omega_{w}$ is a part of $\partial \Omega$ corresponding to solid walls.

The volume energy takes into account energies of each phase with stable states found for $\varphi= \pm 1$ and the contribution due to interfaces. According to the pioneer work of van der Waals ${ }^{45}, f_{m}(\varphi, \nabla \varphi)$ is written as

$$
f_{m}(\varphi, \boldsymbol{\nabla} \varphi)=\frac{\lambda}{4 \zeta^{2}}\left(\varphi^{2}-1\right)^{2}+\frac{\lambda}{2}\|\boldsymbol{\nabla} \varphi\|^{2},
$$

for which $\lambda$ is an energy by unit of length. Remark that the first term of the right-hand side of (4) is the Ginzburg-Landau doublewell potential with two local minima in $\varphi= \pm 1$. The wall energy $f_{w}(\varphi)$ takes into account the energy due to the triple line created between the two phases in contact with a solid wall. In this case, the geometry of the interface has to verify a physical 


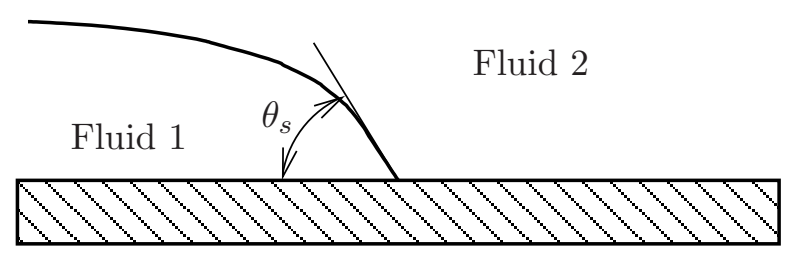

Wall

FIGURE 1 Sketch of the triple line between phases 1 and 2 and the wall. The static contact angle is written $\theta_{s}$.

requirement as shown in FIGURE 1. According to the Young-Dupré law, the static contact angle $\theta_{s}$ is given by ${ }^{46}$

$$
\cos \theta_{s}=\frac{\sigma_{w 2}-\sigma_{w 1}}{\sigma}
$$

for which $\sigma$ is the surface tension between the two phases, $\sigma_{w 1}$ the surface energy between the phase 1 and the wall and $\sigma_{w 2}$ the surface energy between the phase 2 and the wall.

The wall energy describing the wetting physics in a static equilibrium state is given by the relation ${ }^{44}$

$$
f_{w}(\varphi)=-\sigma \cos \theta_{s} \frac{\varphi\left(3-\varphi^{2}\right)}{4}+\frac{\sigma_{w 1}+\sigma_{w 2}}{2},
$$

The equilibrium state is obtained when the functional $\mathcal{F}[\varphi]$ reaches a minimum. The variational derivative ${ }^{47}$ of $\mathcal{F}[\varphi]$ allows to define two quantities

$$
\begin{array}{r}
\mu(\varphi)=\lambda\left[\frac{\varphi\left(\varphi^{2}-1\right)}{\zeta^{2}}-\nabla^{2} \varphi\right], \forall x \in \Omega, \\
L(\varphi)=\lambda \boldsymbol{\nabla} \varphi \cdot \boldsymbol{n}-\frac{3\left(1-\varphi^{2}\right) \sigma}{4} \cos \theta_{s}, \forall x \in \partial \Omega_{w},
\end{array}
$$

for which equation (7) defines the chemical potential in the bulk and equation (8) the wall chemical potential ${ }^{28}$. In the equilibrium state, both (7) and (8) have to be equal to zero.

In one dimension case where the interface is defined on $x=0$, the equilibrium phase field is obviously given by the following solution

$$
\varphi(x)=\tanh \left(\frac{x}{\sqrt{2 \zeta}}\right) .
$$

Moreover, the macroscopic surface tension between the two fluids already introduced above is defined by ${ }^{31}$

$$
\sigma=\lambda \int_{-\infty}^{\infty}\left(\frac{d \varphi}{d x}\right)^{2} d x
$$

which gives with the solution (9)

$$
\sigma=\frac{2 \sqrt{2}}{3} \frac{\lambda}{\zeta}
$$

This last equation is very important since it links a macroscopic property $\sigma$ and microscopic quantities $\lambda$ and $\zeta$.

For vanishing inertia, the fluid dynamics has to be described thanks to the Stokes equations in which the source term due to interfaces must be introduced. The generalization of the Cahn-Hilliard equations coupled to the Navier-Stokes equations is described by the "model H" according to the nomenclature of Hohenberg and Halperin ${ }^{48}$. In the case of incompressible fluids, the model takes the following form

$$
\begin{aligned}
\boldsymbol{\nabla} \cdot \boldsymbol{u} & =0, \\
-\boldsymbol{\nabla} P+\boldsymbol{\nabla} \cdot[2 \eta(\varphi) \boldsymbol{D}(\boldsymbol{u})]+\rho(\varphi) \boldsymbol{g}+\mu \boldsymbol{\nabla} \varphi & =0, \\
\frac{\partial \varphi}{\partial t}+\boldsymbol{\nabla} \varphi \cdot \boldsymbol{u} & =\boldsymbol{\nabla} \cdot[M(\varphi) \boldsymbol{\nabla} \mu(\varphi)], \\
\mu(\varphi) & =\frac{\lambda}{\zeta^{2}}\left[\varphi\left(\varphi^{2}-1\right)-\zeta^{2} \boldsymbol{\nabla}^{2} \varphi\right],
\end{aligned}
$$

where $\boldsymbol{u}$ is the velocity field, $\boldsymbol{P}$ the pressure taking into account the part of the chemical potential deriving from a potential. Equation (12) describes the mass conservation for which each fluid is assumed incompressible. Equation (13) corresponds to 
the momentum balance. The second term of the left-hand side of (13) is the viscous stress for which $\boldsymbol{D}(\boldsymbol{u})$ is the rate-of-strain tensor given by

$$
\boldsymbol{D}=\frac{1}{2}\left(\boldsymbol{\nabla} \boldsymbol{u}+{ }^{t} \boldsymbol{\nabla} \boldsymbol{u}\right) .
$$

The third term of (13) is the volume force due to gravity in which $\boldsymbol{g}$ is the gravitational acceleration and the last term of (13) is the capillary force related to the chemical potential and the gradient of the order parameter. A demonstration of the momentum equation in the framework of phase field formulation can be found in ${ }^{49}$.

Equation (14) is the Cahn-Hilliard equation which has to be coupled to the last equation (15) corresponding to the chemical potential. The quantity $M(\varphi)$ is the Onsager mobility.

Equations (12-15) have to be completed by initial and boundary conditions. For the kinematic quantities, the no-slip conditions will be used on boundaries considered as solid walls. Natural (Neumann) boundary conditions setting the normal stress will be also used depending on the problem at hand. For the chemical potential, the variational formulation of the Cahn-Hilliard equation gives the natural boundary condition ${ }^{29}$

$$
\frac{\partial \mu}{\partial n}=0, \forall x \in \partial \Omega,
$$

meaning that the flux of the chemical potential is equal to zero on the boundary.

For the order parameter, if we assume that the equilibrium is reached on the wall meaning that equation (8) is equal to zero at any time, the boundary condition writes

$$
\lambda \boldsymbol{\nabla} \varphi \cdot \boldsymbol{n}=\frac{3\left(1-\varphi^{2}\right) \sigma}{4} \cos \theta_{s}, \forall \boldsymbol{x} \in \partial \Omega_{w} .
$$

At the interface between the two fluids, i.e. when $\varphi=0$, the directional derivative of $\varphi$ with an outward normal $\boldsymbol{n}$ is proportional to the cosine of $\theta_{s}$. Outside of interface, the boundary condition on $\varphi$ is reduced to an homogeneous Neumann condition. According to molecular dynamics computations achieved by Matsumoto et al. ${ }^{50}$, the time scale of the equilibrium of the wetting at a solid wall is the order of nanosecond justifying the assumption used at the wall.

In the following, the Stokes/Cahn-Hilliard equations are written under dimensionless form with a characteristic length $L$, velocity $U$, viscosity $\eta_{1}$ and density $\rho_{1}$ which will be precised as a function of the problem at hand presented in section 5 . The Onsager mobility is assumed constant. The chemical potential is reduced using the ratio $\lambda / \zeta^{2}$. The time is normalized by $L / U$. The dimensionless coupled system of equations is given by

$$
\begin{aligned}
\boldsymbol{\nabla} \cdot \boldsymbol{u} & =0, \\
-\boldsymbol{\nabla} P+\boldsymbol{\nabla} \cdot[2 \eta(\varphi) \boldsymbol{D}(\boldsymbol{u})]+\frac{\mathrm{Bo}}{\mathrm{Ca}} \rho(\varphi) \boldsymbol{g} & +\frac{3}{2 \sqrt{2} \mathrm{CaCn}} \mu \boldsymbol{\nabla} \varphi=0, \\
\frac{\partial \varphi}{\partial t}+\boldsymbol{\nabla} \varphi \cdot \boldsymbol{u} & =\frac{1}{\mathrm{Pe}} \boldsymbol{\nabla}^{2} \mu(\varphi), \\
\mu(\varphi) & =\varphi\left(\varphi^{2}-1\right)-\mathrm{Cn}^{2} \nabla^{2} \varphi,
\end{aligned}
$$

in which dimensionless variables are written with the same notation as previously. Six dimensionless numbers arise defined as follows

$$
\begin{gathered}
\text { Bo }=\frac{\rho_{1} g L^{2}}{\sigma}, \\
\mathrm{Cn}=\frac{\zeta}{L},
\end{gathered}
$$

$$
\begin{gathered}
\mathrm{Ca}=\frac{\eta_{1} U}{\sigma}, \\
\hat{\rho}=\frac{\rho_{2}}{\rho_{1}},
\end{gathered}
$$

$$
\begin{gathered}
\mathrm{Pe}=\frac{U \zeta^{2} L}{M \lambda}, \\
\hat{\eta}=\frac{\eta_{2}}{\eta_{1}} .
\end{gathered}
$$

The Bond number Bo measures the ratio of gravity to surface tension forces characterized by the surface tension $\sigma$ while the capillary number Ca compares the viscosity effect to the surface tension one. The third dimensionless number Pe is a Péclet number comparing the diffusion time scale of the chemical potential to the convective time scale which is always greater than one. The Cahn number $\mathrm{Cn}$ is the ratio of the diffuse-interface thickness $\zeta$ to the characteristic length scale. The normalized density and dynamic viscosity are given by an arithmetic average:

$$
\begin{aligned}
& \rho(\varphi)=\frac{1+\hat{\rho}}{2}+\frac{1-\hat{\rho}}{2} \varphi, \\
& \eta(\varphi)=\frac{1+\hat{\eta}}{2}+\frac{1-\hat{\eta}}{2} \varphi .
\end{aligned}
$$




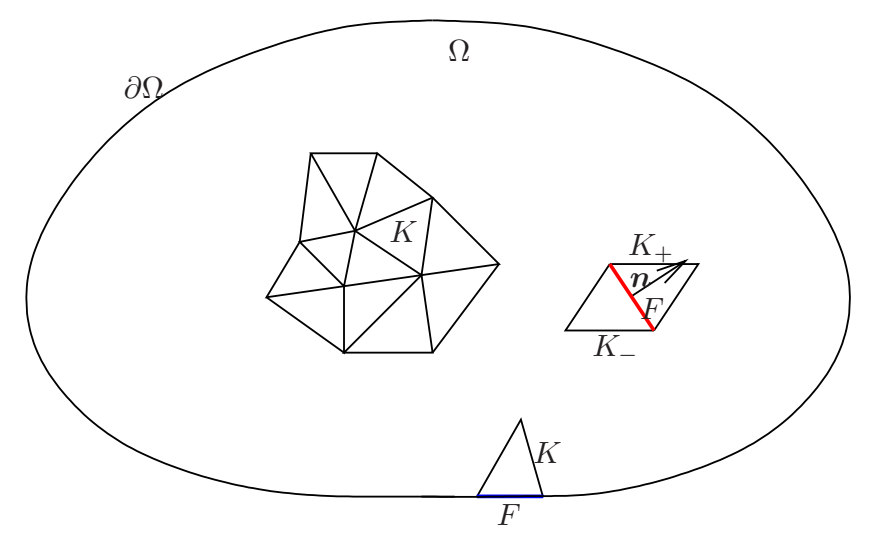

FIGURE 2 Triangulation of the domain $\Omega$ and representation of interior faces in red and boundary faces in blue. The orientation of the interior normal between two triangles $K_{+}$and $K_{-}$is also drawn.

\section{3 | NUMERICAL SOLUTION OF CAHN-HILLIARD EQUATION}

The numerical method is first focused on the Cahn-Hilliard equation which is a non-linear fourth-order partial differential equation needing a high level of regularity. Since the diffusion of the chemical potential is very weak outside interfaces, the parabolic behavior due to the advection term is predominant. Consequently, a discontinuous Galerkin finite element method has been chosen to solve the Cahn-Hilliard equation advected with a known solenoidal velocity field $\boldsymbol{u}$. The time range is $[0, T]$.

\section{1 | Discontinuous Galerkin finite element formulation}

The continuous problem is

Problem 1 (Cahn-Hilliard problem). Find $\varphi(x, t)$ and $\mu(\boldsymbol{x}, t)$ defined in $\Omega \times[0, T]$ such as

$$
\begin{array}{r}
\frac{\partial \varphi}{\partial t}+\boldsymbol{u} \cdot \boldsymbol{\nabla} \varphi-\frac{1}{\mathrm{Pe}} \nabla^{2} \mu(\varphi)=0, \text { in } \Omega \times[0, T], \\
-\mu(\varphi)+\varphi\left(\varphi^{2}-1\right)-\mathrm{Cn}^{2} \nabla^{2} \varphi=0, \text { in } \Omega \times[0, T], \\
\varphi(x, t=0)=\varphi_{0}(\boldsymbol{x}), \text { in } \Omega, \\
\frac{\partial \varphi}{\partial n}=f(\varphi), \text { on } \partial \Omega, \\
\frac{\partial \mu}{\partial n}=0, \text { on } \partial \Omega .
\end{array}
$$

Moreover, the normal component of the velocity $\boldsymbol{u} \cdot \boldsymbol{n}$ is equal to zero on $\partial \boldsymbol{\Omega}$ for $\boldsymbol{n}$ the outward unit normal on $\partial \Omega$. The boundary condition on $\varphi$, eq. (34), has been taken under a general form because in applications the interaction with a wall for instance involves a condition on normal derivative as presented in previous section. The boundary condition on the chemical potential corresponds to the natural condition arisen from the variational form of equation (31) as it has been mentioned in $\S 2$.

A discontinuous Galerkin method initially developed by Kay et al. ${ }^{40}$ has been chosen to solve this problem. Let $\mathcal{T}_{h}$ be a triangulation of the domain $\Omega$ formed by finite elements $K$ with meshsize $h=\operatorname{diam}(K)$ such as $\mathcal{T}_{h}=\{K\}$ and $\bar{\Omega}=\bigcup_{K \in \mathcal{T}_{h}} \bar{K}$ as illustrated in FIGURE 2. Furthermore, we consider a subset of faces $F$ formed by interfaces between two distint mesh elements $K_{+}$and $K_{-}$such as $F=\partial K_{-} \cap \partial K_{+}$represented in red in FIGURE 2 and boundary faces (in blue in FIGURE 2) given by $F=\partial K \cap \partial \Omega$. For internal face, the unit normal $\boldsymbol{n}$ is outward of $K_{-}$and inward of $K_{+}$as it is shown in FIGURE 2. If $\mathcal{F}_{h}^{i}$ gathers all interfaces and $\mathcal{F}_{h}^{b}$ all boundary faces, the set of faces is $\mathcal{F}_{h}=\mathcal{F}_{h}^{i} \cup \mathcal{F}_{h}^{b}$. Finally, the local length scale at the face $F$ noted $h_{F}$ is defined according to Di Pietro and Ern ${ }^{36}$, chap. 4 page 125.

In the formulation below, some usual notations in the context of the discontinuous Galerkin method have to be presented. First, the broken gradient $\boldsymbol{\nabla}_{h}$ is defined by ${ }^{36}$

$$
\left(\nabla_{h} \varphi_{h}\right)_{\mid K}=\boldsymbol{\nabla}\left(\varphi_{h \mid K}\right), \forall K \in \mathcal{T}_{h}
$$


Moreover, at each interior face $F$, let define $\varphi_{h}^{-}=\varphi_{h \mid K_{-}}$the inner value and $\varphi_{h}^{+}=\varphi_{h \mid K_{+}}$the outer value. We define the jump and the average of $\varphi_{h}$ by

$$
\begin{aligned}
\llbracket\left[\varphi_{h}\right] \rrbracket & =\varphi_{h}^{-}-\varphi_{h}^{+}, \\
\left\{\left\{\varphi_{h}\right\}\right\} & =\frac{\varphi_{h}^{-}+\varphi_{h}^{+}}{2} .
\end{aligned}
$$

The discontinuous finite element space is defined by

$$
X_{h}=\left\{v_{h} \in L^{2}(\Omega) ; v_{h \mid K} \in P_{k}, \forall K \in \mathcal{T}_{h}\right\},
$$

with $k$ the polynomial degree. This space belongs to the broken Sobolev space

$$
H^{1}\left(\mathcal{T}_{h}\right)=\left\{v \in L^{2}(\Omega) ; v_{\mid K} \in H^{1}(K), \forall K \in \mathcal{T}_{h}\right\} .
$$

The variational formulation of the Cahn-Hilliard problem takes the following form ${ }^{40}$

Problem 2. Find $\varphi_{h}, \mu_{h} \in X_{h}$ such that

$$
\begin{array}{r}
\operatorname{Pe}^{-1} a_{h}\left(w_{h}, \mu_{h}\right)+b_{h}\left(w_{h}, \varphi_{h}\right)=0, \text { for } w_{h} \in X_{h}, \\
-\left(q_{h}, \mu_{h}\right)+\left(q_{h}, \varphi_{h}^{3}-\varphi_{h}\right)+\operatorname{Cn}^{2} a_{h}\left(q_{h}, \varphi_{h}\right)=l_{h}\left(q_{h}, \varphi_{h}\right), \text { for } q_{h} \in X_{h},
\end{array}
$$

in which

$$
\begin{array}{r}
a_{h}\left(w_{h}, \mu_{h}\right)=\int_{\Omega} \boldsymbol{\nabla}_{h} w_{h} \cdot \nabla_{h} \varphi_{h} d V-\sum_{F \in \mathcal{F}_{h}^{i}} \int_{F}\left[\left\{\left\{\boldsymbol{\nabla} \mu_{h} \cdot \boldsymbol{n}_{F}\right\}\right\} \llbracket\left[w_{h}\right]\right]+\left\{\left\{\boldsymbol{\nabla} w_{h} \cdot \boldsymbol{n}_{F}\right\}\right\}\left[\left[\mu_{h}\right]-c_{F} \llbracket\left[\mu_{h}\right]\right]\left[\left[w_{h}\right]\right] d S, \\
\left.b_{h}\left(w_{h}, \varphi_{h}\right)=\left(\frac{\partial \varphi_{h}}{\partial t}, w_{h}\right)+\int_{\Omega} \boldsymbol{\nabla}_{h} \varphi_{h} \cdot \boldsymbol{u}_{h} w_{h} d V+\sum_{F \in \mathcal{F}_{h}^{i}} \int_{F}\left(\frac{1}{2}\left|\boldsymbol{u}_{h} \cdot \boldsymbol{n}_{F}\right|\left[\left[\varphi_{h}\right]\right] \llbracket w_{h}\right]\right]-\boldsymbol{u}_{h} \cdot \boldsymbol{n}_{F} \llbracket\left[\varphi_{h} \rrbracket\left\{\left\{w_{h}\right\}\right\}\right) d S, \\
l_{h}\left(q_{h}, \varphi_{h}\right)=\mathrm{Cn}^{2} \sum_{F \in \mathcal{F}_{h}^{b}} \int f_{h}\left(\varphi_{h}\right) q_{h} d S, \text { for } q_{h} \in X_{h} .
\end{array}
$$

The bilinear form $a_{h}\left(w_{h}, \mu_{h}\right)$ follows from the formulation of the Laplacian operator with the symmetric interior penalty introduced by Arnold ${ }^{51}$ and Castillo ${ }^{52}$ (see for more details Di Pietro and Ern ${ }^{36}$, chap. ${ }^{4}$ ). According to Shahbazi ${ }^{53}$, the penalty parameter $c_{F}$ proportional to $h_{F}^{-1}$ is taken equal to the product $\beta \varpi_{F}$ with $\beta=(k+1)(k+d) / d$ and

$$
\varpi_{F}= \begin{cases}\frac{\operatorname{meas}(\partial K)}{\operatorname{meas}(K)} & \text { for } F \in K \cap \partial \Omega, \\ \max \left[\frac{\operatorname{meas}\left(\partial K_{+}\right)}{\operatorname{meas}\left(K_{+}\right)}, \frac{\operatorname{meas}\left(\partial K_{-}\right)}{\operatorname{meas}\left(K_{-}\right)}\right] & \text {for } F \in K_{-} \cap K_{+} .\end{cases}
$$

The convective term of the phase-field has been written according to the development of Di Pietro and Ern ${ }^{36}$ with an upwinding flux approximation.

For the time discretization, a backward differentiation formula (BDF) at order $p \leq 6$ is used. If $\Delta t$ is the time step, the temporal derivative at the time $t=n \Delta t$ of the $\varphi_{h}^{n}$ at the order $p$ is given by

$$
\frac{\partial \varphi_{h}^{n}}{\partial t}=\frac{1}{\Delta t} \sum_{l=0}^{p} \alpha_{p l} \varphi_{h}^{n-l}+\mathcal{O}\left(\Delta t^{p}\right),
$$

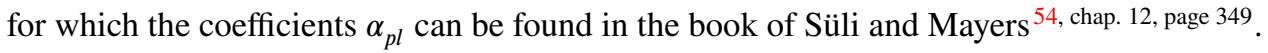

This time derivative determined by the previous formula is introduced in $b_{h}\left(w_{h}, \varphi_{h}^{n}\right)$ leading to a source term obtained with a combination of $\varphi_{h}^{n-l}$ with $1 \leq l \leq p$ following equation (47). Moreover, the non-linearities of the Cahn-Hilliard problem are solved using a damped-Newton algorithm ${ }^{55}$. The scheme guarantees the global convergence for any initial guess. In practice, the BDF scheme is convenient to program and provides good results of conservation apply to an advection equation as it has been applied by Ta et al. ${ }^{35}$ for $p$ equal or greater than 2 . 


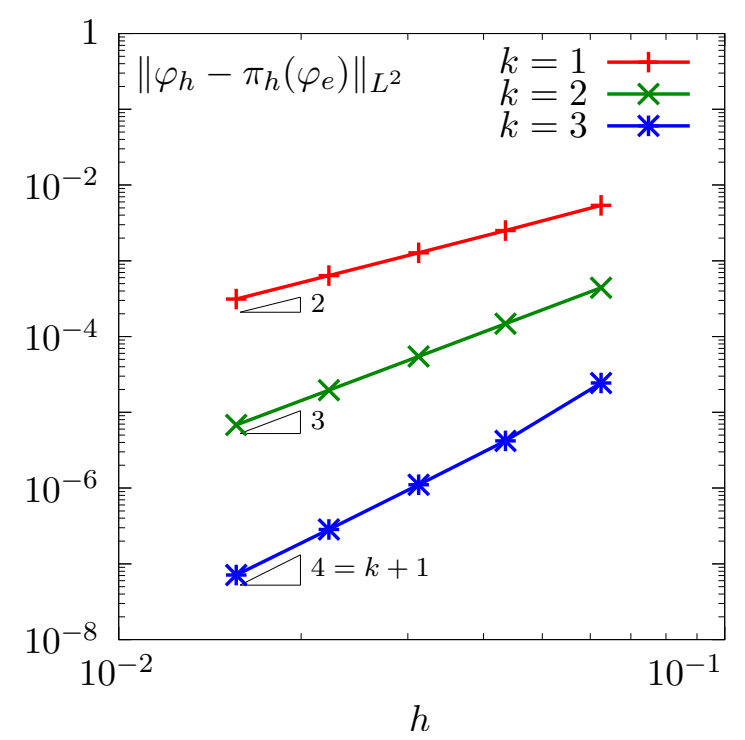

FIGURE 3 Error $\left\|\varphi_{h}-\pi_{h}\left(\varphi_{e}\right)\right\|_{L^{2}}$ as a function of mesh size for the three polynomial degrees.

\section{2 | Spatial convergence rate}

The previous problem has been implemented in the Rheolef $\mathrm{C}++$ finite element library ${ }^{55}$ tested on a numerical problem proposed by Kay et al. ${ }^{40}$. The Cahn-Hilliard equation is solved with a velocity field given by

$$
\begin{array}{r}
u=f(r)(y,-x)^{t}, \forall(x, y) \in[-1 ; 1]^{2}, \\
\text { with } f(r)=\frac{1+\tanh [\beta(1-3 r)]}{2}, \text { and } r=\sqrt{x^{2}+y^{2}},
\end{array}
$$

in a domain $\Omega=[-1 ; 1]^{2}$. This velocity field is a vortex centered in the origin with an amplitude decreasing exponentially with the radial distance from the origin. The exact solution of the phase-field given by

$$
\varphi_{e}=t \cos (\pi x) \cos (\pi y), \forall(x, y) \in[-1 ; 1]^{2} \text { and } t \in[0 ; 1],
$$

is imposed by adding an adequate source term in eq. (31). The range of time is taken in [0;0.1]. The domain has been discretized with a regular triangular elements with 16, 23, 32, 45 and 64 elements over each Cartesian coordinate.

We perform the numerical experiment with Cahn number equal to $10^{-1}$ and for a Péclet number equal to 50. The temporal numerical scheme at the second order (BDF-2) has been used with a time step equal to $10^{-2}$. Three polynomial degrees have been tested with $k=1,2$ and 3. Errors between the numerical solution and the exact solution have been computed with $L^{2}$ norm defined as follows

$$
\|v\|_{L^{2}}=\left(\int_{\Omega} v^{2} d V\right)^{\frac{1}{2}}
$$

FIGURE 3 depicts errors averaged over the time range $[0 ; 0.1]$ as a function of the mesh size both for the phase field and the chemical potential. For each polynomial degree, errors behave as $\mathcal{O}\left(h^{n}\right)$. The exponent $n$ has been determined by non-linear fitting. For $k=1, n$ are equal to 2.08 and 2.00, for $k=2, n=3.01$ and 3.17 and for $k=3, n=4.78$ and 5.18 respectively for $\varphi$ and $\mu$. As expected, errors computed in $L^{2}$ norm behave approximately as $\mathcal{O}\left(h^{k+1}\right)$ both for the phase field $\varphi$ and for the chemical potential $\mu$ showing that the numerical implementation is optimal-order in error bounds as it is proved by Kay et al. ${ }^{40}$.

\subsection{Effect of the Cahn number on mass conservation}

As already mentioned above, the Cahn-Hilliard model conserves the mass over the entire domain. Nevertheless, due to the diffusion over the interface, the mass conservation of each phase is an important issue. According to Jacqmin ${ }^{33}$ and Yue et al. ${ }^{56}$, it is expected that the total free energy defined by equation (3) decreases with the time by reducing the area of interfaces between the two phases leading to a spontaneous formation of spherical inclusions. But the energy can also decrease due to the 
(a) $h=4 \cdot 10^{-2}$

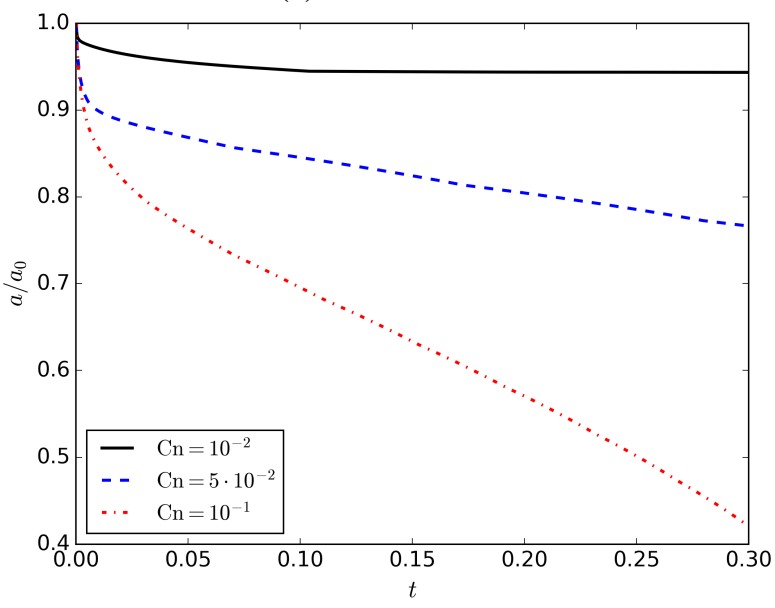

(b) $h=2 \cdot 10^{-2}$

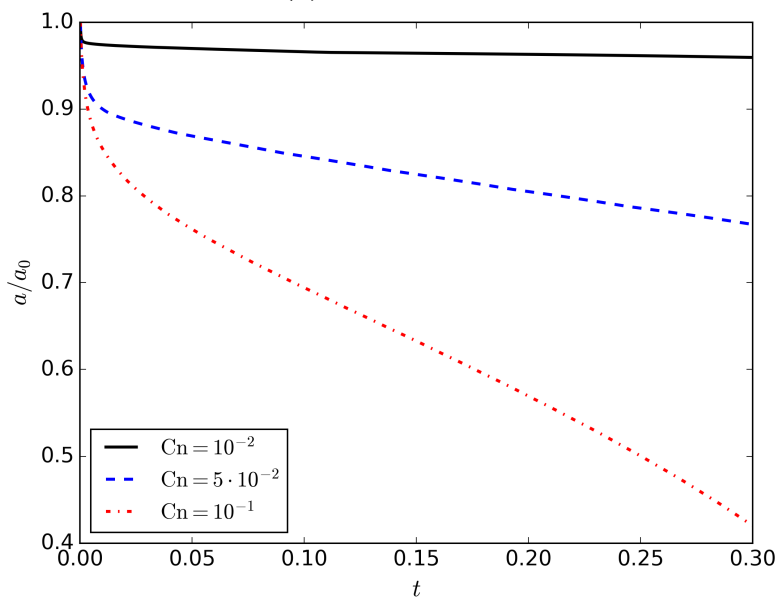

FIGURE $4 a / a_{0}$ as a function of $t$ for three Cahn numbers and mesh size equal to (a) $h=4 \cdot 10^{-2}$ and (b) $h=2 \cdot 10^{-2}$.

shrinkage of drops similar to an Ostwald ripening. Nevertheless, if the order parameter can keep the stable value equal to \pm 1 far away from the interface, the shrinkage can be reduced. Moreover, the Cahn number plays an important role in this dynamics since this number controls the thickness of interfaces. According to Lowengrub and Truskinovsky ${ }^{24}$, the asymptotic limit when Cn goes to zero converges toward a sharp-interface description. Nevertheless, if the Cahn number is too small the Cahn-Hilliard equation becomes too stiff which penalizes strongly the numerical method. So, it is important to find a Cahn number acceptable from the point of view of the numerical solution and also from the point of view of the physics.

To test the effect of the Cahn number on the spontaneous shrinkage of a drop, a simple case has been made in which a static spherical drop with an initial radius equal to $1 / 2$ is introduced in a liquid at rest. Three Cahn numbers have been investigated: $\mathrm{Cn}=10^{-1}, 5 \cdot 10^{-2}$ and $10^{-2}$. The numerical simulations are made in $2 \mathrm{~d}$-axisymmetric geometry for which $(r, z) \in[0,1.5] \times$ $[-1.5,1.5]$. Two mesh sizes have been also tested: a coarse mesh with $h=4 \cdot 10^{-2}$ and a fine mesh with $h=2 \cdot 10^{-2}$.

FIGURE 4 depicts the behavior of the drop radius as a function of time for the three Cahn numbers and for the two mesh sizes. Clearly, the drop shrinks spontaneous when the Cahn number is sufficiently large. The shrinkage rate increases with the Cahn number. This general behavior is shown whatever the mesh size used in the numerical computations. It is noteworthy that the shrinkage is prevented when the Cahn number is equal to $10^{-2}$. We observe a small shrinkage of the drop at the beginning of the simulation mainly due to the fact that initially the chemical potential is not in equilibrium state. This point is a numerical artefact because the initial conditions are only applied on the order parameter $\varphi$.

The absence of drop shrinkage can be explained due to the previous analysis provided by Yue et al. ${ }^{56}$. Indeed, they determined the critical drop size below which drop shinks spontaneous. This critical drop radius normalized by the initial drop size is given by

$$
r_{c}=\sqrt[4]{\frac{2^{1 / 6}}{3 \pi} V \mathrm{Cn}}
$$

in which $V$ is the volume of the computational domain $\Omega$. With $V=27 \pi / 4$, this critical radius is equal to $0.7,0.6$ and 0.4 for $\mathrm{Cn}=10^{-1}, 5 \cdot 10^{-2}$ and $10^{-2}$ respectively. So, for the smallest Cahn number, the initial drop size is larger than the critical radius preventing the spontaneous shrinkage. From this preliminary study, the spontaneous shrinkage is avoided when the Cahn number is less than $10^{-2}$ which is a value acceptable for the numerical scheme. This result confirms the previous conclusion drawn by Yue et al. ${ }^{56}$. The important point to notice is that the numerical domain has not to be too large to prevent the diffusion between phases. This means that the Cahn-Hilliard theory is mainly adapted to mesoscale investigations. According to Yue et al. ${ }^{56}$, the accuracy on the surface tension is proportional to $\mathrm{Cn}^{2}$ and on the mixture properties as the viscosity scale like $\mathrm{Cn}$.

From this numerical test, the Cahn-Hilliard theory can be applied to study two-phase flows when the Cahn number is sufficiently small. A typical value of $10^{-2}$ is enough to prevent the spontaneous shrinkage. Decrease the Cahn number below to $10^{-2}$ leads to a strong stiffness required a high mesh resolution. Consequently, for the numerical applications, $\mathrm{Cn}=10^{-2}$ will be used for a moderate numerical volume to avoid the spontaneous shrinkage. 


\section{4 | NUMERICAL SOLUTION OF CAHN-HILLIARD AND STOKES EQUATIONS}

The continuous problem is similar to the set of equations (19-22) given in $\S 2$. Initial and boundary conditions for $\varphi$ and $\mu$ can be written in general form as in equations (33-35). To take into account the boundary conditions for which velocity or stress are imposed, the boundary of $\Omega$ is shared between $\partial \Omega_{D}$ for which

$$
u=u_{D},
$$

and $\partial \Omega_{N}$ where

$$
\boldsymbol{\sigma} \cdot \boldsymbol{n}=\boldsymbol{t}_{N},
$$

such as $\partial \Omega_{D} \cap \partial \Omega_{N}=\emptyset$ and $\partial \Omega=\partial \Omega_{D} \cup \partial \Omega_{N}$. The stress tensor is given by $-P \boldsymbol{I}+2 \eta(\varphi) \boldsymbol{D}(\boldsymbol{u})$ with $\boldsymbol{I}$ the unit tensor. Boundary faces, $\mathcal{F}_{h}^{b}$, are now shared in two sub-sets $\mathcal{F}_{h}^{b, D}$ and $\mathcal{F}_{h}^{b, N}$ corresponding to $\partial \Omega_{D}$ and $\partial \Omega_{N}$ respectively.

A discontinuous Galerkin finite element method is also used to solve the Stokes equations. As it will be detailed below velocity and pressure are approximated at the same polynomial degree $m$. So, the discontinuous finite element spaces are defined by

$$
\begin{aligned}
& \boldsymbol{V}_{h}=\left\{\boldsymbol{u}_{h} \in L^{2}(\Omega)^{d} ; \boldsymbol{u}_{\mid K} \in P_{m}, \forall K \in \mathcal{T}_{h}\right\}, \\
& Q_{h}=\left\{q_{h} \in L^{2}(\Omega) ; q_{\mid K} \in P_{m}, \forall K \in \mathcal{T}_{h}\right\} .
\end{aligned}
$$

\section{1 | Discontinuous Galerkin finite element formulation of Stokes problem}

Since the Stokes equations are in quasi-steady state, the reference of the time is removed in the following to simplify the notation. The variational formulation follows the method initially introduced by Cockburn et al. ${ }^{57}$ which has been studied theoretically by Di Pietro and $\operatorname{Ern}^{36}$. The heterogeneity of the viscosity needs to generalize the formulation according to the previous developments achieved in heterogeneous diffusion ${ }^{36}$, chap. ${ }^{4}$. The discrete variational formulation writes:

Problem 3 (Stokes problem). Find $\boldsymbol{u}_{h} \in V_{h}$ and $P_{h} \in Q_{h}$ such that

$$
\begin{array}{r}
\alpha\left(\boldsymbol{u}_{h}, \boldsymbol{v}_{h}\right)+\beta\left(\boldsymbol{v}_{h}, P_{h}\right)=\lambda(\boldsymbol{v}), \forall \boldsymbol{v}_{h} \in \boldsymbol{V}_{h}, \\
\beta\left(\boldsymbol{u}_{h}, q_{h}\right)-\gamma\left(p_{h}, q_{h}\right)=0, \forall q_{h} \in Q_{h},
\end{array}
$$

with

$$
\begin{array}{r}
\alpha\left(\boldsymbol{u}_{h}, \boldsymbol{v}_{h}\right)=\int_{\Omega} 2 \eta \boldsymbol{D}_{h}\left(\boldsymbol{u}_{h}\right): \boldsymbol{D}_{h}\left(\boldsymbol{v}_{h}\right) d V+\sum_{F \in \mathcal{F}_{h}^{i} \cup \mathcal{F}_{h}^{b, D}} \int_{F}\left[c_{F} \eta_{F} \llbracket u \rrbracket \cdot \llbracket \boldsymbol{v} \rrbracket-\left\{\left\{2 \eta \boldsymbol{D}_{h}\left(\boldsymbol{u}_{h}\right) \cdot \boldsymbol{n}_{F}\right\}\right\}_{\omega} \llbracket \llbracket \boldsymbol{v}_{h} \rrbracket-\right. \\
\left.\left.\left\{\left\{2 \eta \boldsymbol{D}_{h}\left(\boldsymbol{v}_{h}\right) \cdot \boldsymbol{n}_{F}\right\}\right\}_{\omega} \llbracket\left[\boldsymbol{u}_{h}\right]\right]\right] d S, \\
\beta\left(\boldsymbol{u}_{h}, q_{h}\right)=-\int_{\Omega} q_{h} \boldsymbol{\nabla}_{h} \cdot \boldsymbol{u}_{h} d V+\sum_{F \in \mathcal{F}_{h}^{i}} \int_{F} \llbracket u \rrbracket \cdot \boldsymbol{n}_{F}\left\{\left\{q_{h}\right\}\right\} d S, \\
\lambda(\boldsymbol{v})=\int_{\Omega}\left[\frac{\mathrm{Bo}}{\mathrm{Ca}} \rho_{h} \boldsymbol{g}+\frac{3}{2 \sqrt{2} \mathrm{CaCn}} \mu_{h} \boldsymbol{\nabla}_{h} \varphi_{h}\right] \cdot \boldsymbol{v}_{h} d V+\sum_{F \in \mathcal{F}_{h}^{b, D}} \int_{F} \boldsymbol{u}_{h, D} \cdot\left[c_{F} \boldsymbol{v}_{h}-2 \eta \boldsymbol{D}_{h}\left(\boldsymbol{v}_{h}\right) \cdot \boldsymbol{n}\right] d S+ \\
\sum_{F \in \mathcal{F}_{h}^{b, N}} \int_{F} \boldsymbol{t}_{h, N} \cdot \boldsymbol{v}_{h} d S, \\
\left.\gamma\left(p_{h}, q_{h}\right)=\sum_{F \in \mathcal{F}_{h}^{i}} \int_{h_{F}}\left[p_{h}\right]\right]\left[\left[q_{h} \rrbracket\right] d S .\right.
\end{array}
$$

The viscosity at the face $F$ and the weighted average $\left\{[\cdot\}_{\omega}\right.$ are defined by ${ }^{36}$

$$
\begin{aligned}
& \eta_{F}=\frac{2 \eta_{h}^{+} \eta_{h}^{-}}{\eta_{h}^{+}+\eta_{h}^{-}}, \\
& \left\{\left\{v_{h}\right\}\right\}_{\omega}=\frac{\eta_{h}^{+} v_{h}^{-}+\eta_{h}^{-} v_{h}^{+}}{\eta_{h}^{+}+\eta_{h}^{-}} .
\end{aligned}
$$

Clearly, when the viscosity is constant, the usual arithmetic average is found. 


\section{2 | Algorithm of Cahn-Hilliard/Stokes solver}

The algorithm developed to solve the coupled Cahn-Hilliard/Stokes problem is based on the sequential steps. First remark that as for pressure field, the chemical potential does not need initial solution. Nevertheless, a particular caution is needed on the initial chemical potential field to avoid numerical instabilities due to a non smooth solution of $\mu$. While the initialization of the order parameter is easy to do, the chemical potential is not known. To initiate the phase-field solution, we only solve the Cahn-Hilliard equation without motion over few hundreds of time steps before to solve the full problem.

Now, consider that at the time step $n$, the fields $\varphi_{h}^{n}, \mu_{h}^{n}, \boldsymbol{u}_{h}^{n}$ and $P_{h}^{n}$ are known, the algorithm is decomposed as follows

1. Determination of $\varphi_{h}^{n+1}, \mu_{h}^{n+1}$ by solving Problem 2 using a BDF- $p$ scheme for which the velocity field is extrapolated at the same order that the $\mathrm{BDF}$ scheme using the forward difference formula ${ }^{58}$ :

$$
\boldsymbol{u}_{h}^{*, n}=\sum_{l=1}^{p}\left(\begin{array}{l}
p \\
l
\end{array}\right)(-1)^{l-1} \boldsymbol{u}_{h}^{n-l}+\mathcal{O}\left(\Delta t^{p}\right) .
$$

2. Determination of $\boldsymbol{u}_{h}^{n+1}$ and $P_{h}^{n+1}$ by solving Problem 3 in which the capillary source term given by the last term of the right-hand side of (20) is determined by the previous solution of $\varphi_{h}^{n+1}, \mu_{h}^{n+1}$.

This procedure is reproduced until the final time of the computation. To control the rates of convergence of our algorithm, a test of comparison with an exact solution is described below.

\subsection{Spatial and time rates of convergence}

To control spatial and time rates of convergence of our numerical algorithm, we enforce a body source term in momentum and Cahn-Hilliard equations to impose an exact solution. The problem is considered in a two-dimensional space with $\boldsymbol{x}=(x, y) \in$ $[0 ; 1]^{2}$. According to the previous test of Dong and Shen ${ }^{59}$, the exact phase-field solution is taken as follows

$$
\varphi(x, t)=\cos (\pi x) \cos (\pi y) \sin (t) .
$$

Using the definition of the chemical potential, the exact solution of $\mu$ is

$$
\mu(\boldsymbol{x}, t)=\varphi\left(\varphi^{2}+2 \pi^{2} \mathrm{Cn}^{2}-1\right),
$$

with $\varphi$ given by (65).

The two components of the velocity are chosen as follows

$$
\begin{aligned}
& u(x, t)=\sin (\pi x) \cos (\pi y) \sin (t), \\
& v(x, t)=-\cos (\pi x) \sin (\pi y) \sin (t) .
\end{aligned}
$$

Contrary to Dong and Shen ${ }^{59}$, the pressure has not to be specified since $p$ is imposed by the mass conservation. The two source terms which must be added in momentum equation (20) and in Cahn-Hilliard equation (21) are given in Appendix A. Remark that the velocity field is such that the rate-of-strain tensor is a diagonal tensor. The velocity solution is also used as boundary conditions while homogeneous Neumann conditions are imposed both for $\varphi$ and $\mu$.

We made tests without gravity force which is replaced by the source test enforcing the solution. The Cahn number is taken equal to $10^{-1}$, the capillary number is set equal to 1 and the Péclet number at 10 . The viscosity ratio is $\hat{\eta}=10^{-1}$. To control the spatial convergence, five mesh grids have been used with a regular triangle mesh with sizes $h$ equal to $[1 / 16,1 / 23,1 / 32,1 / 45,1 / 64]$ which are uniformly logarithmically spaced. The convergence rate is determined by computing the $L^{2}$ norms both for $\varphi, \mu$ and $\boldsymbol{u}$. The numerical computations are achieved in time range between 0 and $\pi / 2$ with a time step equal to $\pi \cdot 10^{-4}$ and with BDF-2 for the time integration scheme. FIGURE 5-(a) depicts the logarithm of errors both for $\varphi, \mu$ and $\boldsymbol{u}$ as a function of the logarithm of $h$. Polynomial degrees have been set equal to 1 and 2 for both $\varphi, \mu$ and $\boldsymbol{u}$. Whatever the polynomial degree and the field, the error behave as $h^{n}$. A non-linear fitting gives $n=1.94,1.91$ and 1.98 when $k=1$ and 3.93, 3.18 and 3.01 when $k=2$ for $\varphi, \mu$ and $\boldsymbol{u}$ respectively. These results show that errors behaves approximately as $\mathcal{O}\left(h^{k+1}\right)$. The error bounds of our numerical solver is consequently optimal-order in space.

The time rate of convergence is determined on a mesh grid $h=1 / 32$. The Cahn, capillary and Péclet numbers are taken at the same values that previously used for the spatial rate of convergence. The time step ranges from $\pi / 400$ to $\pi / 100$. The final time is set equal at $\pi / 2$. Only, the BDF-2 scheme has been tested. A second order polynomial approximation is taken both for $\varphi, \mu$ and $\boldsymbol{u}$. FIGURE 5-(b) presents the logarithm of errors both for $\varphi, \mu$ and $\boldsymbol{u}$ as a function of the logarithm of $\Delta t$. Temporal 

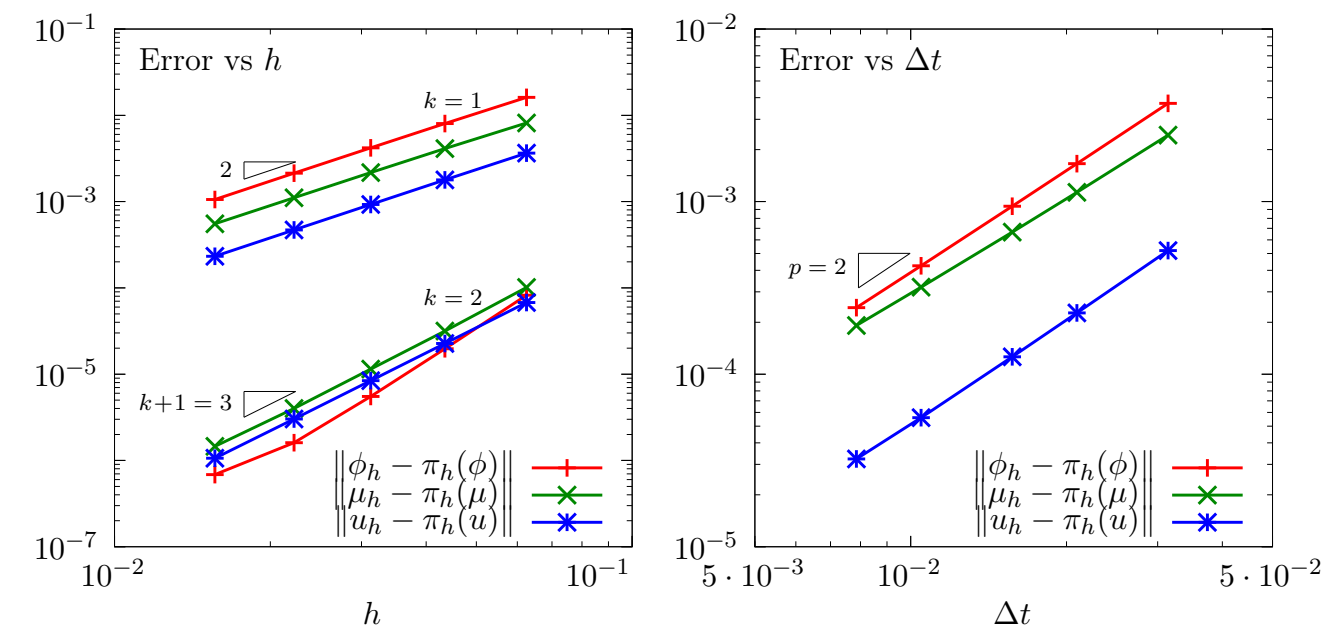

FIGURE 5 Convergence of $\varphi, \mu$ and $\boldsymbol{u}$ as a function of $h$ (a) and $\Delta t$ (b).

errors behave as $\Delta t^{n}$. A non-linear fitting gives $n=1.98,1.86$ and 2.04 for $\varphi, \mu$ and $u$ respectively meaning that the second order is verified.

\section{4 | Mesh adaptation}

The numerical scheme introduces a mesh adaptation loop in order to capture the interface with a better spatial resolution. The main principle of the auto-adaptive mesh follows the procedure provided by Castro-Diaz et al. ${ }^{60}$. The criterion used here is based on the total energy variation. Indeed according to Jacqmin ${ }^{33}$ it can be shown that the temporal derivative of the total energy is given by

$$
\frac{D E}{D t}=-\int_{\Omega}\left[2 \eta(\varphi) \boldsymbol{D}(\boldsymbol{u}): \boldsymbol{D}(\boldsymbol{u})+\frac{1}{\mathrm{Pe}} \boldsymbol{\nabla} \mu(\varphi) \cdot \nabla \mu(\varphi)\right] d V .
$$

The criterion is then chosen to well describe the dissipation of the total energy.

From an initial mesh, a solution is computed. The criterion is given by

$$
c=2 \eta(\varphi) \boldsymbol{D}(\boldsymbol{u}): \boldsymbol{D}(\boldsymbol{u})+\frac{1}{\mathrm{Pe}} \boldsymbol{\nabla} \mu(\varphi) \cdot \boldsymbol{\nabla} \mu(\varphi),
$$

which depends also on the polynomial approximations taken both by $\boldsymbol{u}$ and $\mu$. If the polynomial degrees are different, a projection is proceeded. The mesh adaptation procedure uses the anisotropic mesh generator BAMG ${ }^{61}$. The strategy is based on a metric determined from the Hessian of the scalar $c$ given by (70). The procedure is repeated at any time which required an interpolation step between two successive time steps.

We postpone the numerical examples in the next section showing the mesh adaptation.

\section{5 | NUMERICAL RESULTS ON TWO-PHASE FLOWS}

In this section, three numerical problems are presented. The first case considers the drop retraction in a liquid at rest, the second will be carried out on the capillary rising of a liquid to see the interaction with wall. The last case will be devoted to the wetting dynamics of a drop on a horizontal solid wall.

\section{1 | Ellipsoidal droplet retraction}

A non-spherical droplet composed by a fluid with a dynamic viscosity $\eta_{2}$ carried in another fluid at rest with a dynamic viscosity $\eta_{1}$ backs to the spherical shape to reduce the surface energy. This phenomena is fundamental to find the constitutive equation 


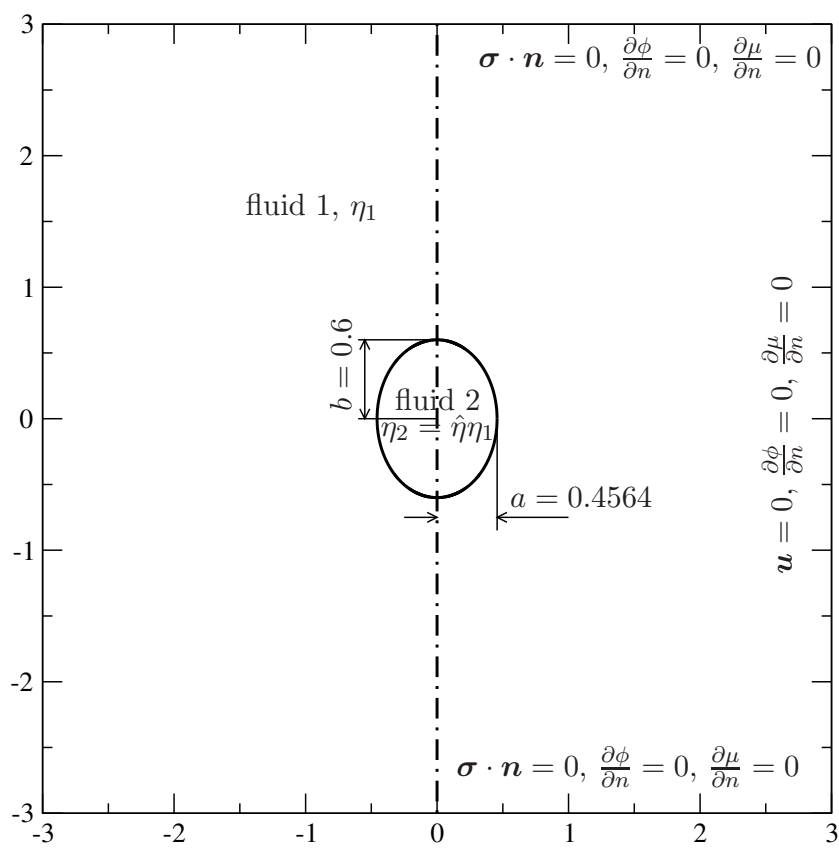

FIGURE 6 Initial configuration of an ellipsoidal droplet with a major axis equal to 0.6 and a minor axis equal to 0.4564 . Boundary conditions both for $\varphi, \mu$ and $\boldsymbol{u}$ are also indicated.

of emulsion ${ }^{62}$. If the droplet is lightly deformed and in the limit of vanishing Reynolds number, the characteristic sizes of the droplet behave exponentially with time ${ }^{63}$.

In this problem the gravity is removed meaning that the Bond number is an irrelevant dimensionless number for this particular case. Since only the capillary number is involved in this physics, the velocity scale is chosen in order to set the capillary number equal to one. Consequently, the velocity scale is obviously equal to $\sigma / \eta_{1}$. Only the viscosity ratio $\hat{\eta}$ remains as dimensionless number from the point of view of the physics.

Beside, the Cahn and the Péclet numbers related to the phase-field modelling have to be specified. The physical length scale of interface is around few nanometers meaning that the ratio of this interface thickness and the droplet size is too small. Fortunately, the "sharp-interface" behavior is obtained for a larger Cahn number according the previous work of Yue et a. ${ }^{31}$. Moreover, we previously shown in subsection 3.3 that the reduction of free energy due to the Cahn-Hilliard equation becomes negligible when the Cahn number becomes equal to $10^{-2}$. Consequently, the Cahn number is set equal to $10^{-2}$. Moreover, to reduce the effect of the diffusion between the two phases, the Onsager mobility can be chosen sufficiently low meaning that the Péclet number can be taken greater than one. Here, the Péclet number is taken equal to $10^{2}$.

Initially, the droplet shape is considered as an ellipse with a minor axis $a$ and a major axis $b$. The characteristic time scale is given by $2 a_{\infty} \eta_{1} / \sigma$ in which $a_{\infty}$ is the radius of the drop obtained in steady-state regime observed when the time goes to infinity. The problem is formulated in $2 \mathrm{~d}$-axisymmetric space. The domain $\Omega$ is extended over $r$-axis until a radius equal to 3 . In $z$-axis, the domain is between $z \in[-3,3]$. Obviously, the mass conservation leads to $a_{\infty}=\sqrt[3]{a^{2} b}$ when the axis of revolution is over the major axis. FIGURE 6 presents the initial configuration of the droplet and the boundary conditions on $\varphi, \mu$ and $\boldsymbol{u}$. The major and minor axis have been set equal to 0.6 and 0.4564 respectively in order to have $a_{\infty}=1 / 2$ when the time goes to infinity. From the numerical approximation, a same polynomial degree equal to 2 is used for all unknowns. A BDF-2 scheme is chosen for the time integration. At the boundaries, the no-slip condition is used for the velocity field on the right boundary while on the top and on the bottom frontiers, stress free condition is used to reduce the effect of the finite domain. Both on $\varphi$ and $\mu$ fields, homogeneous Neumann condition is imposed. Initially, the velocity field is set equal to zero and the phase-field solution is initialized using the method indicated at the beginning of this section.

The deformation of the droplet remains moderate to compare with the asymptotic solution obtained from Frankel and Acrivos ${ }^{63}$. When the deformation is weak, the square difference between the major and the minor axis are given according to 
(a) $t=0$

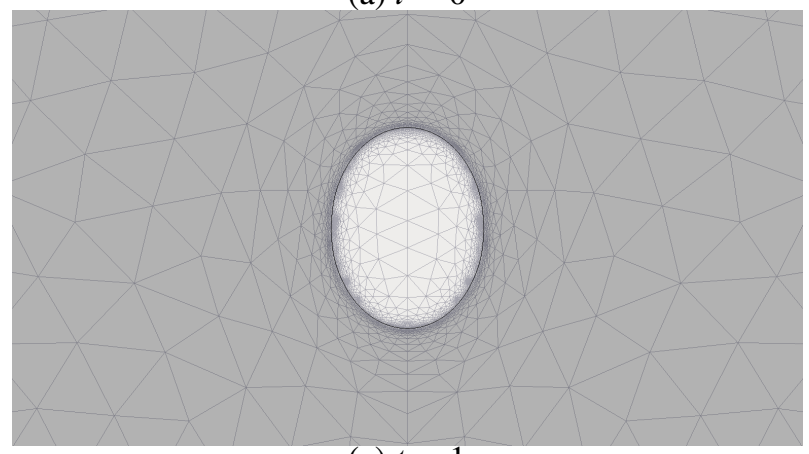

(c) $t=1$

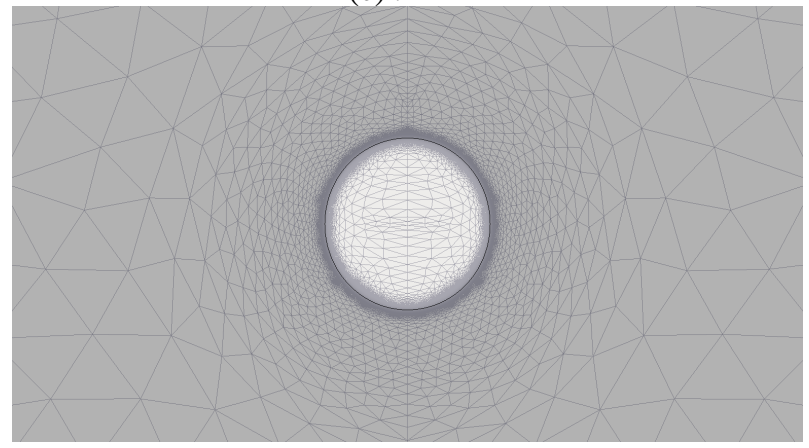

(b) $t=0.5$

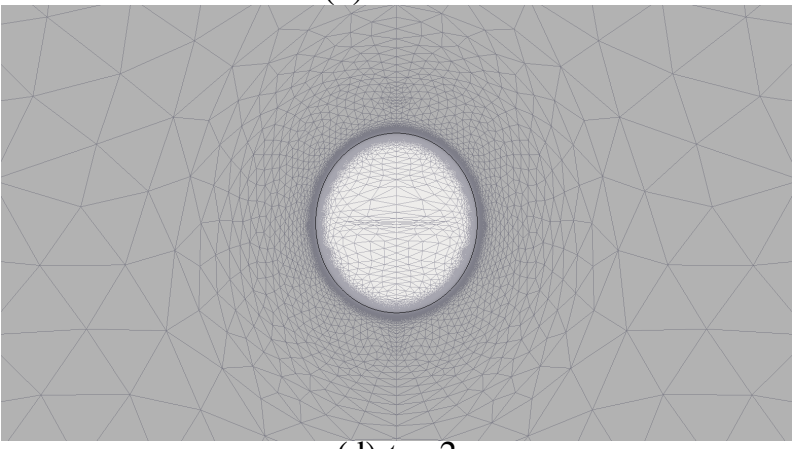

(d) $t=2$

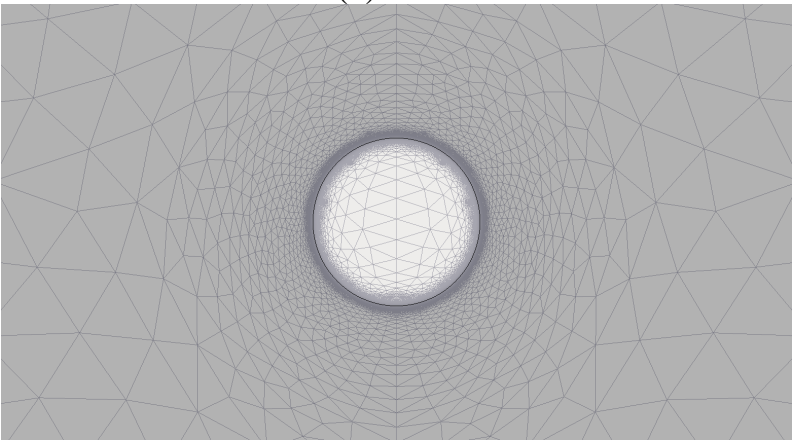

FIGURE 7 Order parameter $\varphi$ and mesh grids around the drop during the viscous retraction with $\hat{\eta}=10^{-2}$ at the time (a) $t=0$, (b) $t=0.5$, (c) $t=1$ and (d) $t=2$.

Frankel and Acrivos ${ }^{63}$ and Maffetone and Minale ${ }^{64}$ by the following solution

$$
\frac{\left(b^{2}-a^{2}\right)(t)}{\left(b^{2}-a^{2}\right)(0)}=\exp [-f(\hat{\eta}) t]
$$

with

$$
f(\hat{\eta})=\frac{80(1+\hat{\eta})}{(3+2 \hat{\eta})(16+19 \hat{\eta})}
$$

and $t$ in dimensionless unit.

Two numerical simulations have been made with a viscosity ratio equal to $10^{-2}$. The first computation is performed on a fix mesh grid with a mesh size equal to $1.4 \cdot 10^{-2}$. The second computation has been done with a mesh adaptation loop with the criterion defined in subsection 4.4. To illustrate the mesh adaptation procedure, mesh grids obtained during the numerical procedure are provided in FIGURE 7 with the order parameter field. At the time $t=0$, the mesh is already adapted due to the pre-computation of the Cahn-Hilliard equation. Three other snapshots are depicted in FIGURE 7 at (b) $t=0.5$, (c) $t=1$ and (d) $t=2$. The finest mesh size is set equal to $2.5 \cdot 10^{-3}$ while the largest mesh size is equal to $1 / 2$. Obviously, the finest meshes are concentrated over the interface between the two fluids.

FIGURE 8 shows the behavior of $\left(b^{2}-a^{2}\right)(t) /\left(b^{2}-a^{2}\right)(0)$ as a function of time for $\hat{\eta}=10^{-2}$ both for the numerical simulations performed without and with mesh adaptation. These solutions are compared with the approximate solution given by equation (71) showing that the numerical computations are in perfect agreement with the asymptotic solution.

At the end of numerical runs, the droplet should be perfectly spherical with a radius equal to $1 / 2$. To control this asymptotic behavior, the pressure is plotted over $r$-and $z$-axis directions in FIGURE 9 for the numerical run achieved without mesh adaptation. The Laplace's law is very well verified since the pressure jump is equal to 4 as expected since the capillary number is equal to 1 and the radius is equal to $1 / 2$. The two curves plotted over the two orthogonal direction show that the good isotropy of the solution. Nevertheless, the two curves are not perfectly superimposed. It is mainly due to the non-compliance of the mirror symmetric over the $r$-axis imposed by the physics. Indeed, to be perfectly symmetric the velocity field due to the retraction should verify the mirror symmetry over the $r$-axis. However, due to residual imperfections in the mesh the symmetry is not exactly fullfiled numerically explaining why the curves are not completely superimposed. It is noteworthy that the disagreement stays small. 


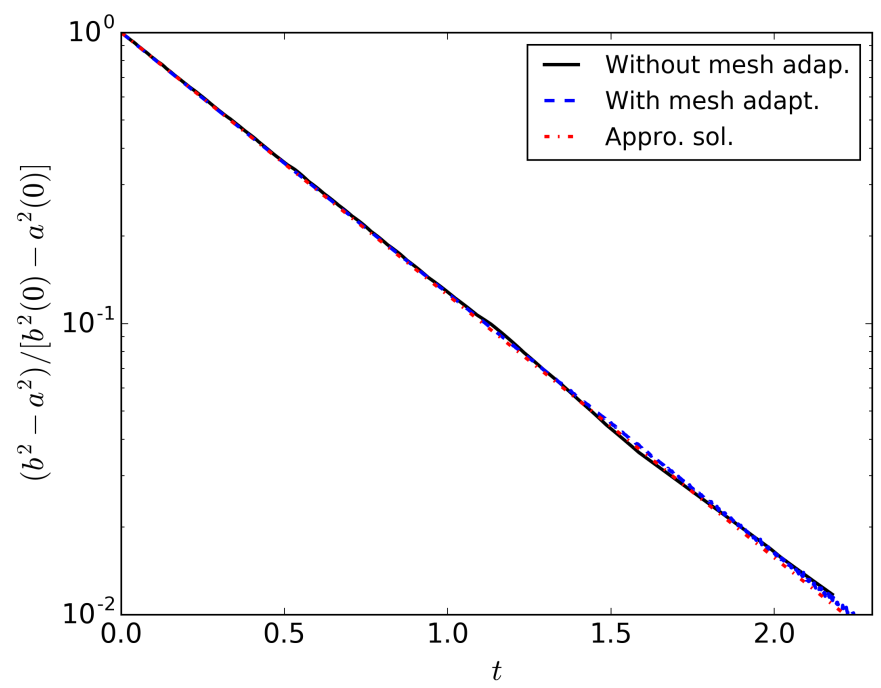

FIGURE $8\left(b^{2}-a^{2}\right)(t) /\left(b^{2}-a^{2}\right)(0)$ vs. $t$ for $\hat{\eta}=10^{-2}$ obtained numerically without mesh adaptation (solid line) and with mesh adaptation (dashed line). Dotted line curve is the approximative solution given by eq. (71).

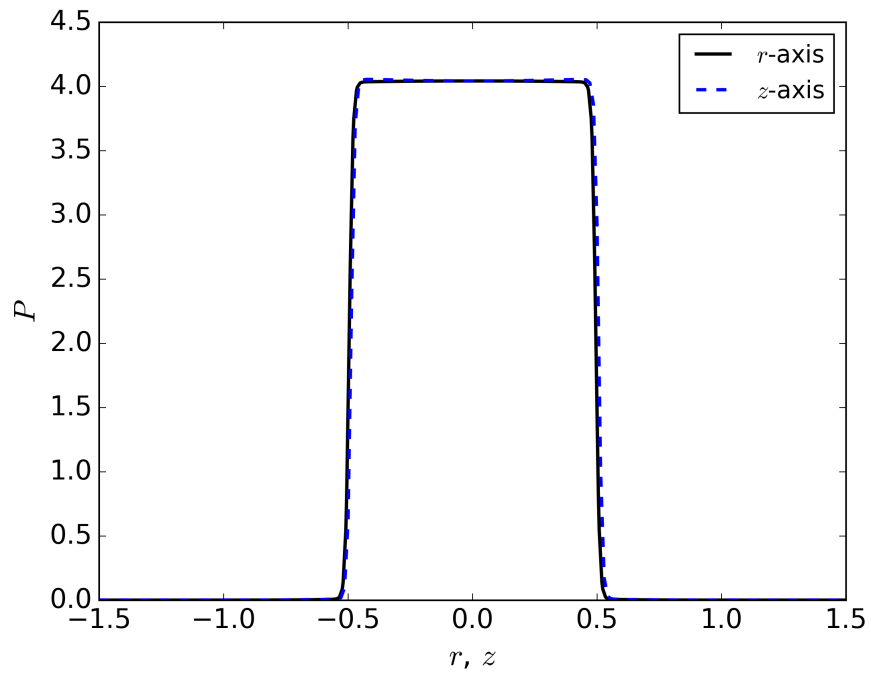

FIGURE $9 p$ vs. $r$ or $z$ for $\hat{\eta}=10^{-2}$ at the end of the numerical run without mesh adaptation.

Numerical computations have been also made for other viscosity ratios. FIGURE 10 presents the time behavior of $\left(b^{2}-\right.$ $\left.a^{2}\right)(t) /\left(b^{2}-a^{2}\right)(0)$ as a function of time obtained numerically for five viscosity ratios $\hat{\eta}$ equal to $10^{-3}, 10^{-2}, 10^{-1}, 1$ and 10 . The exponential decrease is very well captured numerically for all viscosity ratios. Due to a decrease of the retraction rate when the viscosity ratio is large, the time to observe a decrease of $\left(b^{2}-a^{2}\right)(t) /\left(b^{2}-a^{2}\right)(0)$ by two orders of magnitude must be increased. While for $\hat{\eta}=10^{-3}$, a total time equal to 2.5 is enough, it is required that the total time must be around 20 for $\hat{\eta}=10$. Note also that the results for the two smaller viscosity ratio are approximately similar corresponding to the limit for consider the fluid inclusion as a bubble. To perform the numerical integration, the time step is taken equal to $10^{-3}$ when the viscosity ratio is smaller than 1 and $\Delta t=10^{-2}$ for $\hat{\eta}=10$. The comparison with equation (71) shows that the role playing by the viscosity of the droplet is well established even if the numerical solution is found in a finite domain while the approximate solution has been established in an infinite domain. Nevertheless, remark that the rate of retraction given by the logarithmic time derivatives of $\left(b^{2}-a^{2}\right)(t) /\left(b^{2}-a^{2}\right)(0)$ obtained numerically are slightly smaller than the prediction given by eq. (72). This result comes from the effect of the finite domain used in the numerical simulation. Indeed, as it is well-known in Stokes flow, the decreasing of the 


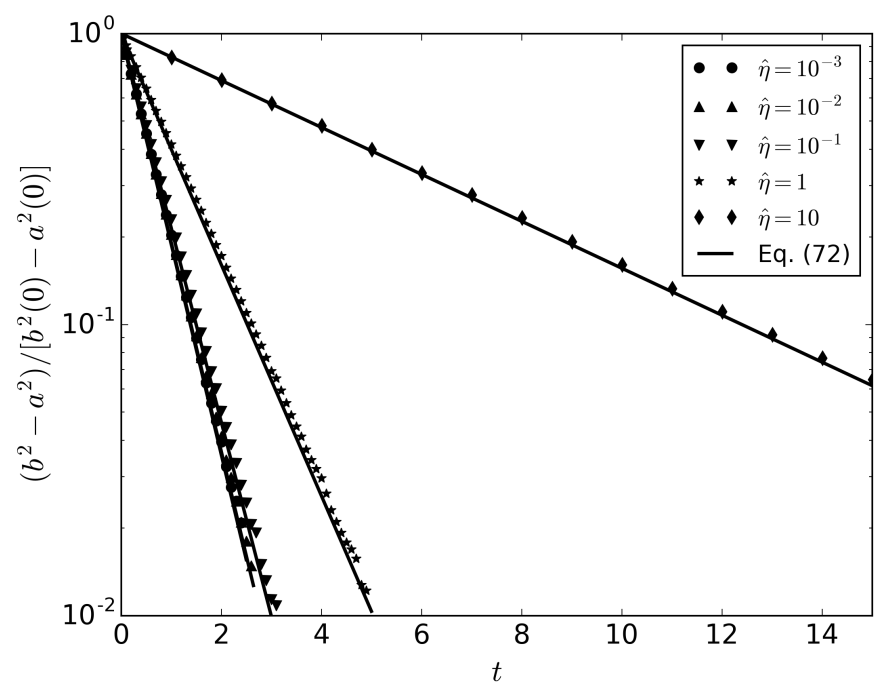

FIGURE $10\left(b^{2}-a^{2}\right)(t) /\left(b^{2}-a^{2}\right)(0)$ vs. $t$ for $\hat{\eta}=10^{-3}, 10^{-2}, 10^{-1}, 1$ and 10 . Solid lines are the numerical results and dashed lines come from the approximative solution given by eq. (71).

flow is very low in space. The fundamental solution of the Stokes flow due to the source point decreases as $1 / r$ with $r$ the radial distance from the source point ${ }^{1}$. So, it is expected with a finite domain simulation to see this effect since the no-slip boundary condition is imposed at a finite distance.

In subsection 3.3 the mass conservation has been controlled for a stationary droplet in a liquid at rest. Here we perform a similar test but for fluids in motion. Moreover, only the numerical runs performed with mesh adaptation loop are presented. To control the mass conservation, the relative difference between the droplet volume at each time and the initial droplet volume is plotted in FIGURE 11 as a function of time divided by the maximum of the time duration of the numerical run for the five viscosity ratios. The volume of the droplet is determined by the relation $4 \pi a^{2} b / 3$. The worst case is observed for the viscosity ratio equal to $\hat{\eta}=10^{-1}$ for which the difference of the droplet volume reaches $2.5 \%$ at the end of the computation. Nevertheless, this difference stays limited while the number of time steps is around $10^{4}$ for all computations. Moreover, to be more in agreement with the fluid dynamics at low Reynolds number, the computation domain has been increased in such a way that the criterion given in subsection 3.3 is not fulfilled. Due to the mesh adaptation, the mass transfer stays limited. Conversely to the results of subsection 3.3, the drop volume increases with time mainly due to the interpolation between meshes required during the mesh adaptation. Despite the change of drop volume which stays limited, the retraction rate is in agreement with the theoretical prediction whatever the viscosity ratio.

\section{2 | Capillary rising}

When a liquid is introduced in a capillary tube with a diameter $D$, the liquid rises up due to the wetting of the liquid on the tube wall. At the equilibrium, the rising height depends on the static contact angle $\theta_{s}$, the surface tension $\sigma$, the liquid density $\rho_{1}$, the gravity and the tube diameter. According to de Gennes et al. ${ }^{46}$, the height over which the liquid rises up can be written as follows

$$
\frac{h_{\text {cap }}}{D}=\frac{4 \cos \theta_{s}}{\text { Bo }},
$$

in which the Bond number is defined with $D$ as a length scale. When $\theta_{s}<\pi / 2$ corresponding to "wetting" condition, $h_{\text {cap }}$ is positif while if $\theta_{s}>\pi / 2$ ("non-wetting" condition), $h_{\text {cap }}$ is negatif meaning that the liquid goes down occurring for a liquid metal like mercury, for instance.

The dynamics of rising can be studied with the Stokes/Cahn-Hilliard equations for which the wetting condition can be introduced easily. We made a numerical simulation in an axisymmetric geometry depicted in FIGURE 12 corresponding to the one half of the tube. The problem is normalized by a length scale equal to the tube diameter. The velocity scale is taken by writing the balance between gravity and viscous forces which gives $U=\rho_{1} g D^{2} / \mu_{1}$. In this case, the capillary and Bond numbers become similar. 


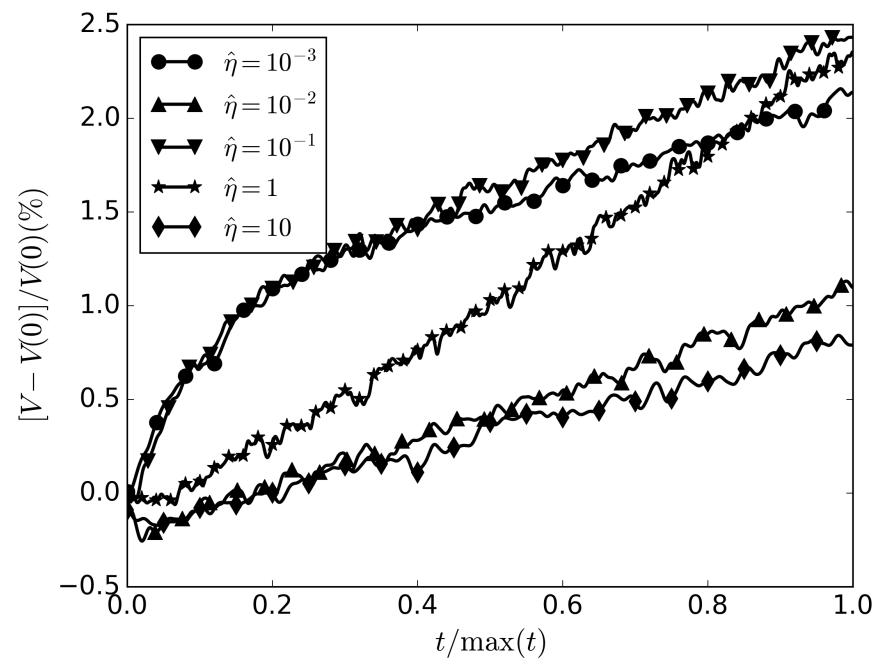

FIGURE $11[V(t)-V(0)] / V(0)(\%)$ vs. $t$ obtained numerically with mesh adaptation for $\hat{\eta}=10^{-3}, 10^{-2}, 10^{-1}, 1$ and 10 .

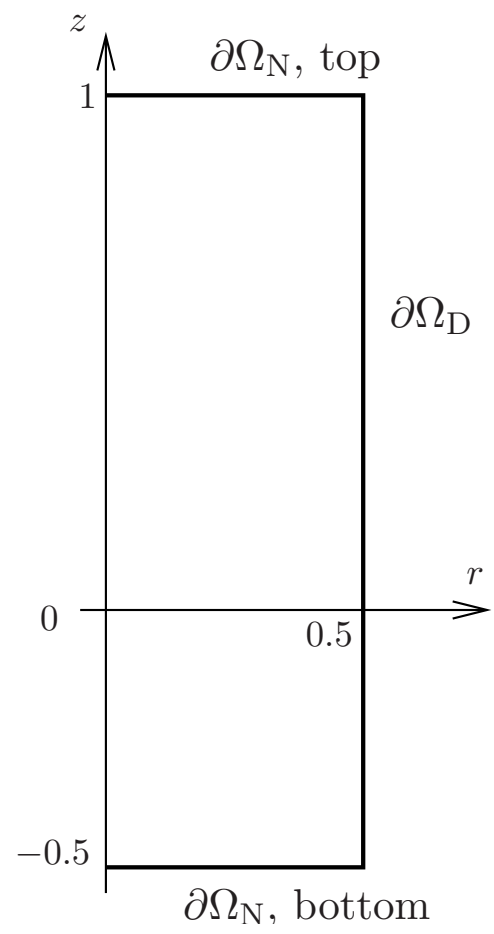

FIGURE 12 Geometry of a liquid rising in a tube with a radius equal to $1 / 2$.

In the limit of vanishing Reynolds number, five dimensionless numbers have to be considered. In the previous test, the "sharpinterface" limit was very well captured when the Cahn number is an order to $10^{-2}$. Consequently, the Cahn number is set equal to $10^{-2}$. The Péclet number has to be taken sufficiently large to reduce the diffusion. The Péclet number Pe is set equal to 50. Ratios $\hat{\rho}$ and $\hat{\eta}$ have been chosen equal to $10^{-3}$ and $10^{-2}$ respectively which are the typical values for water (fluid 1) and air (fluid 2).

The frontier of the domain depicted in FIGURE 12 is composed by a bottom frontier localized at one radius below the initial position of the free surface. In this boundary, a pressure is imposed corresponding to the hydrostatic pressure determined with the initial position of the free surface. By this way, the bottom frontier is similar to a permeable boundary simulating the contact with a reservoir. The vertical boundary which is at one diameter above the initial free surface is an open boundary in which the 


\begin{tabular}{cccc} 
Boundary & $\varphi$ & $\mu$ & $\boldsymbol{u}$ \\
\hline$\partial \Omega_{\mathrm{N}}$, bottom & $\frac{\partial \varphi}{\partial n}=0$ & $\frac{\partial \mu}{\partial n}=0$ & $\boldsymbol{\sigma} \cdot \boldsymbol{n}=-\left(\hat{\rho}+\frac{1}{2}\right) \boldsymbol{n}$ \\
\hline$\partial \Omega_{\mathrm{N}}$, top & $\frac{\partial \varphi}{\partial n}=0$ & $\frac{\partial \mu}{\partial n}=0$ & $\boldsymbol{\sigma} \cdot \boldsymbol{n}=0$ \\
\hline$\partial \Omega_{\mathrm{D}}$ & $\frac{\partial \varphi}{\partial n}=\frac{\left(1-\varphi^{2}\right) \sqrt{2} \cos \theta_{s}}{2 \mathrm{Cn}}$ & $\frac{\partial \mu}{\partial n}=0$ & $\boldsymbol{u}=0$ \\
\hline
\end{tabular}

TABLE 1 Boundary conditions for the numerical computation of the capillary rising of a liquid for the geometry given in FIGURE 12.

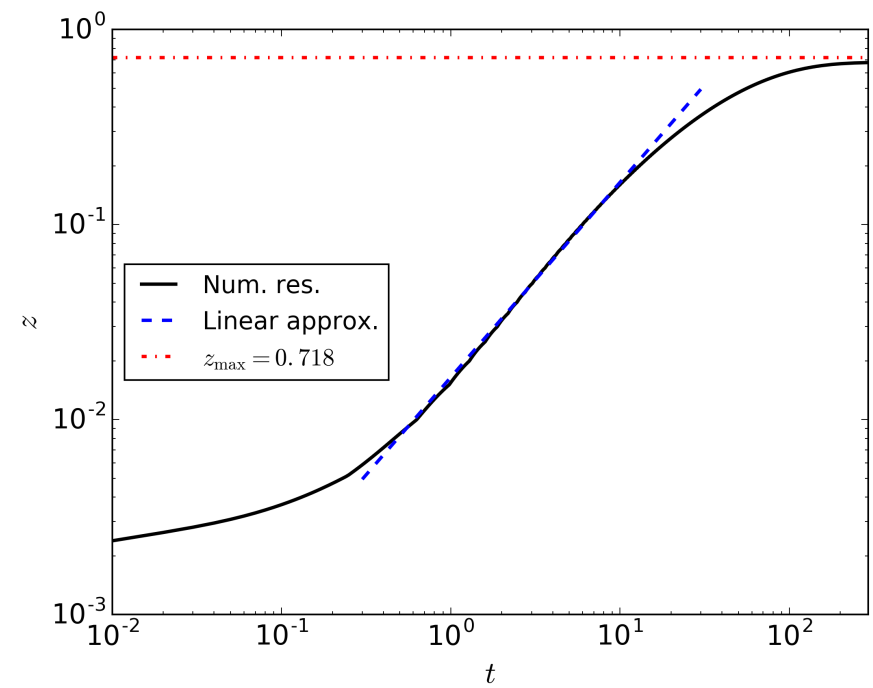

FIGURE 13 Contact line position as a function of time for a contact angle equal to $4 \pi / 9$ and Bo $=1$.

pressure is imposed equal to zero. From the nature of these two conditions, both the bottom and top horizontal boundaries are designated as $\partial \Omega_{\mathrm{N}}$ in FIGURE 12. The vertical frontier $\partial \Omega_{\mathrm{D}}$ localized at $r=1 / 2$ corresponds to the wall in which the no-slip boundary condition is imposed on the velocity field. The whole conditions for both $\varphi, \mu$ and $\boldsymbol{u}$ are summarized in TABLE 1 .

On $\partial \Omega_{\mathrm{D}}$, the wetting condition has been written according to the previous developments summarized in section 2 . By this way, at the free surface corresponding to $\varphi=0$, the wetting angle is imposed through a non-homogeneous Neumann condition on the order parameter. Initially, fluid 1 is located below the plane $z=0$ in such of way that $\varphi$ is given by the exact solution of the Cahn-Hilliard equation in one-dimension:

$$
\varphi_{0}(z, r)=-\tanh \left(\frac{z}{\sqrt{2} \mathrm{Cn}}\right) .
$$

Numerical simulations have been done with BDF-2 temporal scheme and a time step equal to $10^{-3}$. The typical mesh size is roughly equal to $10^{-2}$. Starting with a plane interface, a static contact angle equal to $\theta_{s}=4 \pi / 9\left(80^{\circ}\right)$ is imposed for a Bond number equal to one. The position of the contact line is recorded at each time step. FIGURE 13 presents the position of the contact line as a function of time. The solid line is the numerical solution while the dashed-dotted line is the linear approximation. After a short time over which the static contact angle is established, the contact line rises up quasi-linearly as a function of time. When the free surface is close to the equilibrium position, the motion of the contact line becomes slower and slower. The dashed line corresponds to the position of the contact line which must be observed for $\theta_{s}=4 \pi / 9$ and $\mathrm{Bo}=1$.

Physically speaking, when the contact angle is established on the wall, the liquid below the free surface undergoes a pressure not balanced by the weight of the liquid. Consequently, the liquid rises up until the liquid counterweights the capillary force. In FIGURE 14, the pressure field is plotted at the beginning $t=1$ and at the end of the numerical simulation, $t=288$. In FIGURE 14-(a) recorded at the beginning of the numerical run, a low pressure is mainly observed close to the contact line. An 
(a) $t=1$

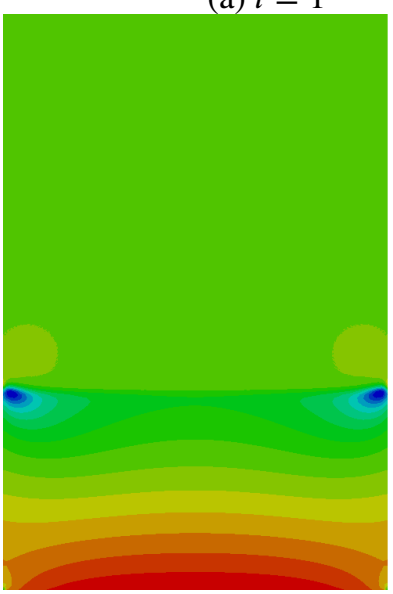

(b) $t=288$

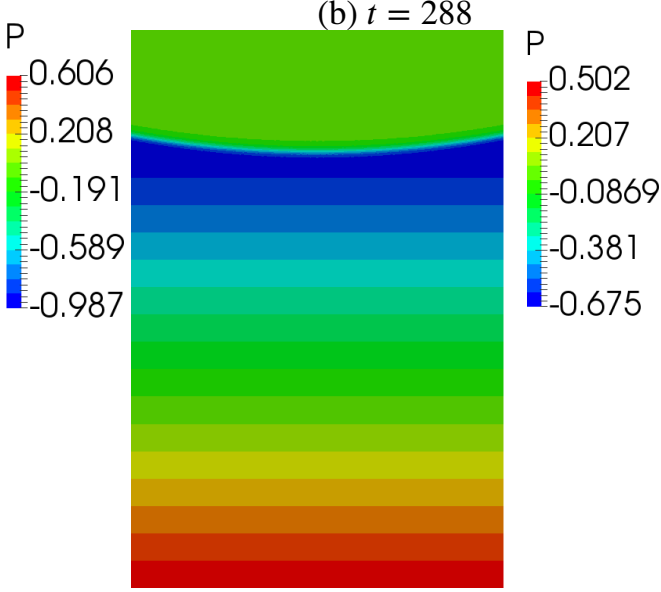

FIGURE 14 Pressure field in the tube at (a) the beginning of the numerical run $t=1$ and (b) at the end $t=288$ for a contact angle equal to $\theta_{s}=4 \pi / 9$ and $\mathrm{Bo}=1$.

over-pressure appears at the bottom of the tube. In FIGURE 14-(b) taken at the end of the numerical simulation, a hydrostatic pressure is fulfilled characterized by a curved free surface.

The position of the free surface after the rising of the liquid can be obtained easily by solving the equilibrium of the pressure due to the gravity and surface tension. If the slope of the free surface is assumed enough small, the free surface is obviously solution of the following equation

$$
\frac{1}{r} \frac{d}{d r}\left(r \frac{d z}{d r}\right)=\text { Bo } z
$$

The left-hand side is the curvature given by the Laplacian of the free surface. The right-hand side is the gravity force. This last equation has been normalized for which only the Bond number is involved. The exact solution is given by

$$
z=C I_{0}(\sqrt{\mathrm{Bo}} r),
$$

for which, $I_{0}$ is the modified Bessel function at the zeroth order. The constant $C$ is determined by setting the contact angle equal to $\theta_{s}$ at the wall position (in $r=1 / 2$ ).

FIGURE 15 plots the $z$ position at the function of $r$ for the free surface obtained numerically at the end of the numerical run. The solution obtained according to the static equilibrium, Eq. (76), is also represented. The agreement between the two solutions is quite good meaning that the static condition is fulfilled.

The Euclidean norm of the velocity field is represented in FIGURE 16 at three different times (1, 25 and 100). Far away of the free surface, velocity profile is similar to a Poiseuille flow driven by the capillary rising. As it is clearly shown in FIGURE 16, the amplitude of the velocity decreases with the time. When $t=0.1$, the maximum of the Euclidean norm is approximately $4.5 \cdot 10^{-2}$ while at $t=25 \max (\|\boldsymbol{u}\|)$ is equal to $1.71 \cdot 10^{-2}$ to reach $2.87 \cdot 10^{-3}$ at $t=100$. Close to the contact line, a strong velocity gradient is observed for which the maximum of the velocity is reached close to the contact line. Nevertheless, due to the diffuse interface, the velocity remains continue.

The behavior of the axial velocity close to the contact line is shown in FIGURE 17 where the $z$-component of the velocity $v$ is plotted as a function of radial coordinate. As already pointed out, the velocity strongly increases close to the contact line mainly due to the pressure gradient driven by the capillary force. The scale over which the velocity changes is due to the diffusion length of the chemical potential coupling with the viscous dissipation. According to Jacqmin ${ }^{27}$ and Yue et al. ${ }^{31}$, it is expected that the length scale of diffusion is proportional to $1 / \sqrt{\mathrm{Pe}}$. In contrary, Briant and Yeomans ${ }^{65}$ proposed another scaling of the diffusion length in $1 / \sqrt[4]{\mathrm{Pe}}$.

To control the prediction of our numerical solver, we made two supplementary runs with $\mathrm{Pe}=10^{2}$ and $10^{3}$. Velocity profiles have been extracted over the radial axis localized at the contact line at a particular time equal to 2.5. FIGURE 18 depicts the behavior of $v / \max (v)$ as a function of $\tilde{x}$ defined as follows

$$
\tilde{x}=\frac{(1-2 r) \sqrt[4]{\mathrm{Pe}}}{2}
$$




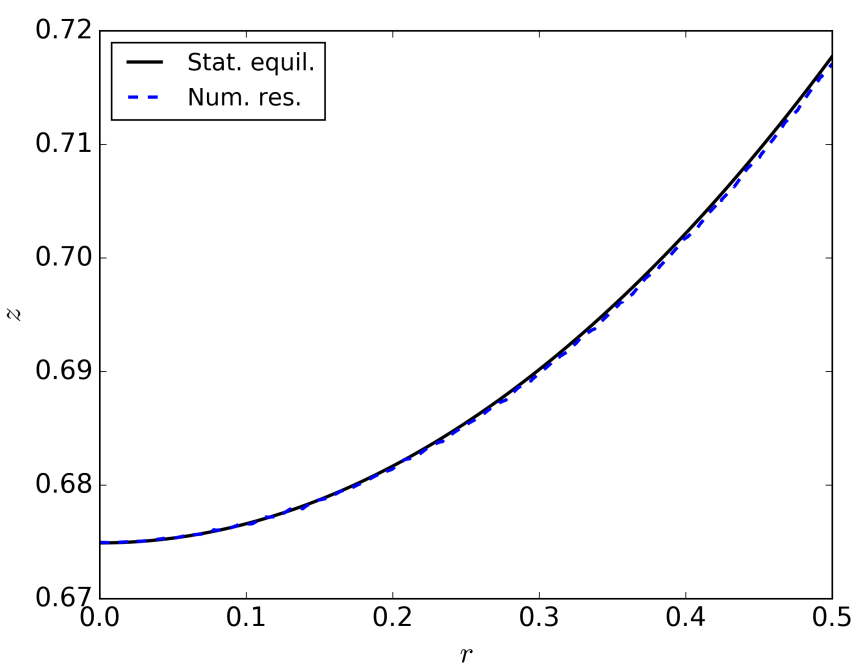

FIGURE 15 Geometry of the free surface, $z$ vs. $r$, for a contact angle equal to $4 \pi / 9$ and Bo $=1$. Solid line is the static equilibrium given by Eq. (76) and dashed-dotted line is the numerical solution.

(a) $t=1$

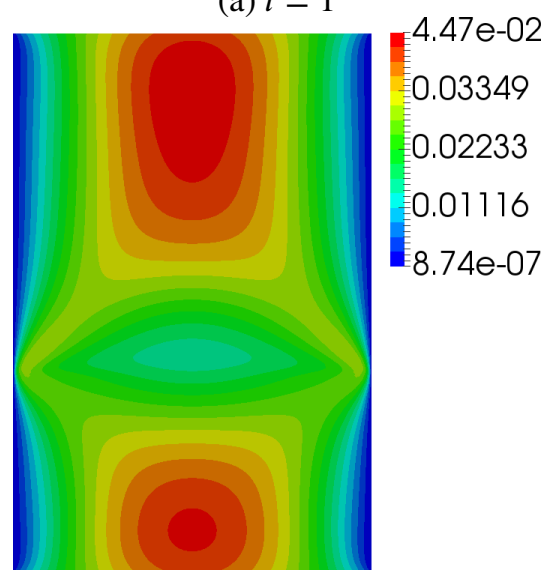

(b) $t=25$

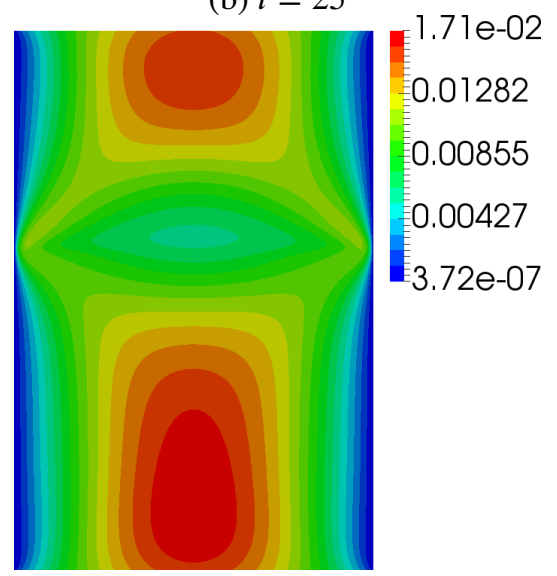

(c) $t=10^{2}$

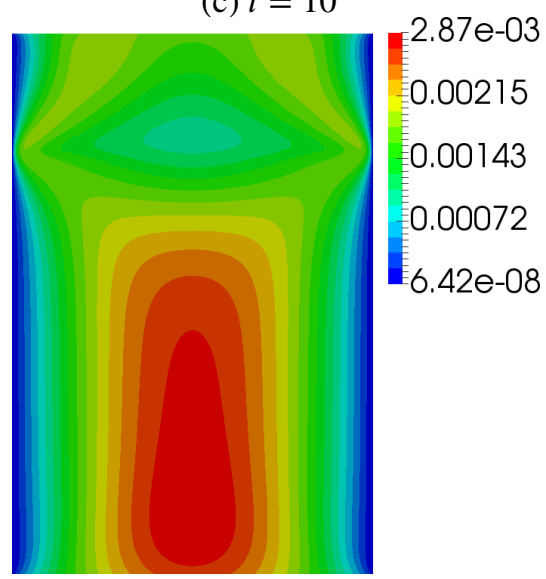

FIGURE 16 Magnitude of the velocity field $\|u\|$ in the tube at (a) $t=1$, (b) $t=25$ and (c) $t=10^{2}$ for a contact angle equal to $4 \pi / 9$ and $\mathrm{Bo}=1$. At each time, the velocity range is rescaled and is given in each figure on the left side.

in the situation where $\mathrm{Bo}=1, \mathrm{Cn}=10^{-2}$. The coordinate $\tilde{x}$ is the inner coordinate according to van Dyke ${ }^{66}$. Clearly, the scaling proposed by Briant and Yeomans ${ }^{65}$ agrees very well with our numerical results. In the reference ${ }^{65}$, the contact line dynamics is driven by a shear flow between two parallel walls while here the driven force is due to the wetting of the liquid. Consequently, our result is a confirmation of the scaling proposed by Briant and Yeomans ${ }^{65}$.

\section{3 | Drop spreading on a horizontal wall}

The last case investigated in this section is devoted to a drop wetting on a horizontal solid substrate. In a such case, and when the gravity force is neglected, a drop at rest takes a static form equivalent to a spherical cap with a static contact angle depending on the wetting property ${ }^{67}$ as already indicated at the beginning of the previous subsection. If now a droplet is set on the substrate with an initial contact angle $\theta_{0}$ different to the static angle $\theta_{s}$, the droplet spreads or retracts depending on the difference $\theta_{0}-\theta_{s}$. More accurately, if $\theta_{0}-\theta_{s}>0$, the drop spreads. Conversely, the drop retracts if $\theta_{0}-\theta_{s}<0$. A numerical simulation has been achieved for the first situation. We artificially put a semi-spherical drop with a dimensionless radius equal to $1 / 2$. In the limit where inertia and gravity are neglected, the only two forces involve in this problem are the viscous and the surface tension 


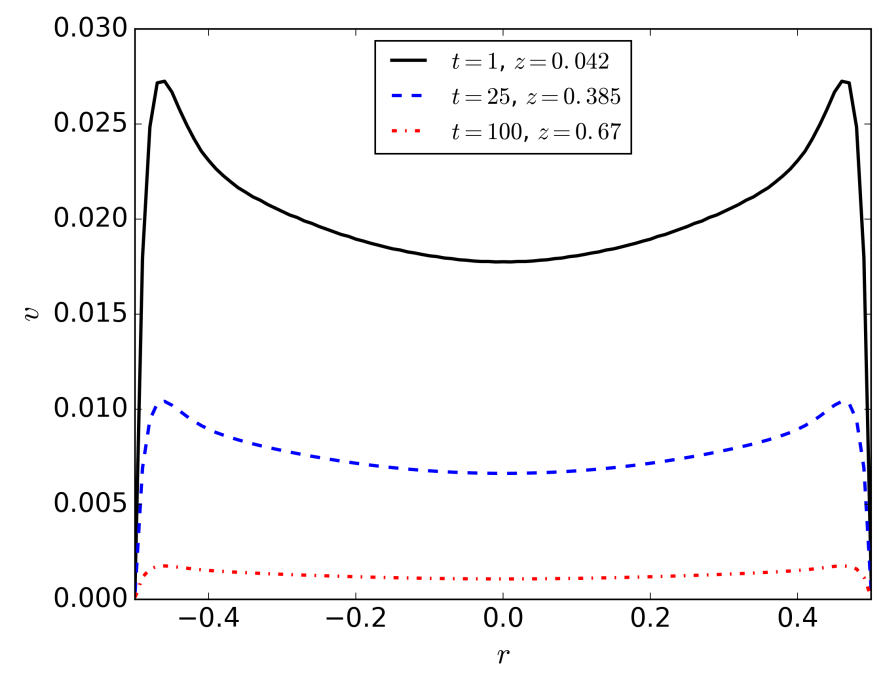

FIGURE $17 z$-axis velocity component $v$ vs. $r$, over a horizontal line localized right on the contact line for a contact angle equal to $4 \pi / 9$ and $\mathrm{Bo}=1$ and for $t=1,25$ and 100 .

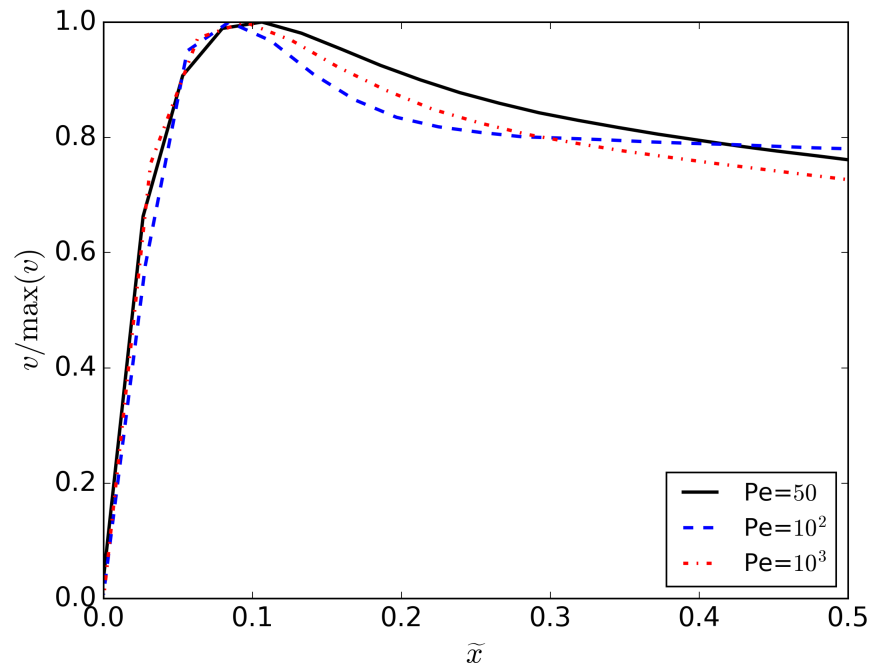

FIGURE $18 v / \max (v)$ as a function of $\widetilde{x}$ at the contact line for $t=2.5$ for $\mathrm{Bo}=1$ and $\mathrm{Pe}=50,10^{2}$ and $10^{3}$.

forces. It is natural in a such limit to take as a characteristic velocity the viscous-capillary velocity given by $\sigma / \eta_{1}$. This means that the only dimensionless numbers are the Cahn and the Péclet numbers and the static contact angle $\theta_{s}$. As previously, the problem is written in a $2 \mathrm{~d}$-axisymmetric geometry in which $(r, z) \in[0,1.5] \times[0,1]$.

The domain is depicted in FIGURE 19 with a drop of the fluid 1 represented at the initial time meaning that the initial contact angle is $\pi / 2$. The boundary conditions are also provided in FIGURE 19 . On the top and the right boundaries, stress free condition is imposed and homogeneous Neumann condition is applied both on $\varphi$ and $\mu$. On the bottom, the no-slip boundary condition is used. The wetting condition is set as previously applied in the case a the capillary rising. The no-flux condition is applied for the chemical potential. The Cahn and the Péclet numbers are taken equal to $10^{-2}$ and $10^{2}$ respectively. The static contact angle is equal to $\pi / 6$. The viscosity ratio has been set equal to $\hat{\eta}=10^{-2}$. The mesh has been made with the finest mesh size close to the wall equal to $1 / 150$ and the largest mesh size far away from the wall is set equal to $1 / 50$. This run has been done without mesh adaptation. The total of triangle elements is then equal to 8617 . The time step is $\Delta t=10^{-3}$ and a BDF-2 scheme is used. 


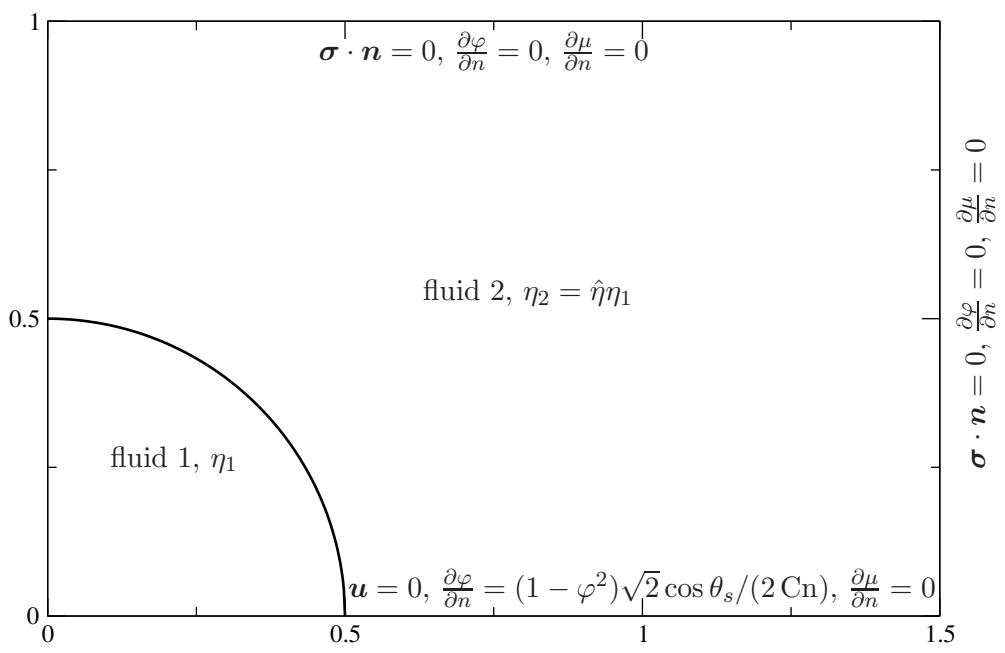

FIGURE 19 Geometry of a spreading drop of fluid 1 in the initial configuration. The boundary condition on the top, bottom and right boundaries are also given for the three unknowns, $\varphi, \mu$ and $\boldsymbol{u}$.

First, the drop shape is represented in solid line in FIGURE 20 at six different times starting from the initial condition $(t=0)$ to a time for which the static form is practically observed, see FIGURE 20-(f). To control the shape of the drop, we approximate the shape by two different curves. The first method is to consider that the drop shape is a spherical cap with a radius $a$, centered on the $z$-axis in $z_{0}$ with a contact angle $\theta$. These three parameters are found by assuming that the drop volume is conserved, and the dimensions of the drop in $r$-axis and $z$-axis are just defined by the geometry which can be written as follows

$$
\begin{array}{r}
a^{3}(1-\cos \theta)^{2}(2+\cos \theta)=\frac{1}{4}, \\
\left.r\right|_{z=0}=a \sin \theta, \\
z_{\text {max }}=a+z_{0} .
\end{array}
$$

In the two last equations, $\left.r\right|_{z=0}$ is the location of the triple line on the wall, i.e. $z=0$ and $z_{\max }$ is the location of the drop interface on the $z$-axis. These two locations can be obtained by the numerical form. The solution of the previous system of equations gives us the three parameters, $a, z_{0}$ and $\theta$. This first approximation will be designated as "global" approximation. The spherical cap obtained by this method is plotted in FIGURE 20 for the different times in dash-dot line.

Another representation of the drop shape has been used. Once again, we consider that the shape can be given by the spherical cap given in a case of acute angle by the following equation

$$
z=z_{0}+\sqrt{a^{2}-r^{2}}
$$

The values of $z_{0}$ and $a$ are determined using a non-linear regression minimizing the error between the numerical and the spherical cap solution. This second approximation designated as "non-linear regression" is plotted in FIGURE 20 in dashed line.

Since for initial condition which is given by a semi-spherical shape, FIGURE 20-(a) and when the drop is close to the static form, FIGURE 20-(f), the two methods give approximately the same curve. A disagreement is more important during the spreading process. In fact, since the static angle is applied as boundary condition, the curvature changes close to the wall which is the main driven force leading to the drop spreading. This change of curvature is particular very well visible in FIGURE 20-(b) and FIGURE 20-(c).

The different between the spherical cap and the real form can be interpreted in term of apparent contact angle. Hoffman ${ }^{68}$ showed experimentally by pushing a liquid in a capillary tube that the apparent contact angle can be different to the static angle. He pointed out also that the difference depends on the velocity or more accurately on the capillary number. In order to describe more rigorously the wetting dynamics, $\operatorname{Cox}{ }^{19}$ develop a theory based an asymptotic development around the contact line. Far away from the contact line, the Stokes equations are used given the fluid dynamics solution. Close to the contact line, Cox introduced a slip length to remove the singularity of the solution at the contact line. Finally, by matching the outer and the inter 
(a) $t=0$

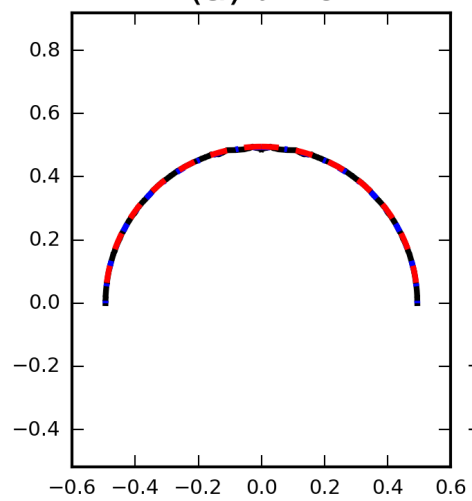

(d) $t=2$

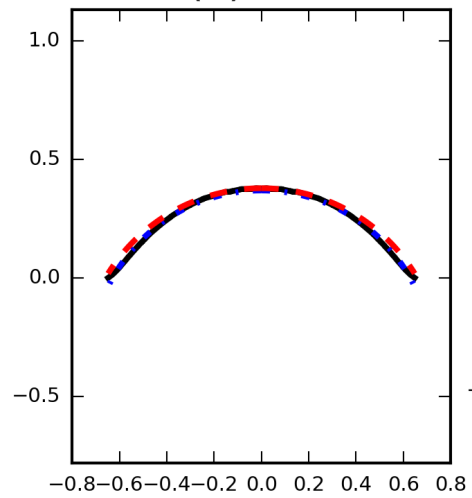

(b) $t=0.1$

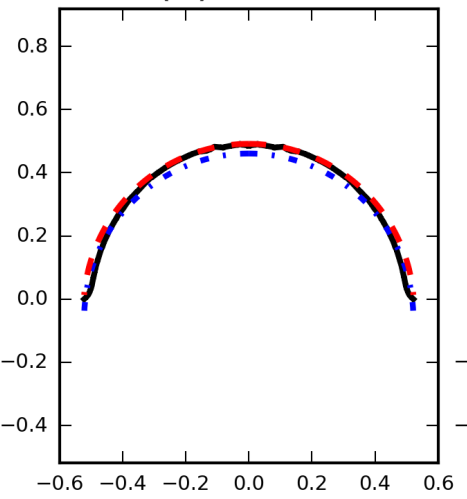

(e) $t=5$

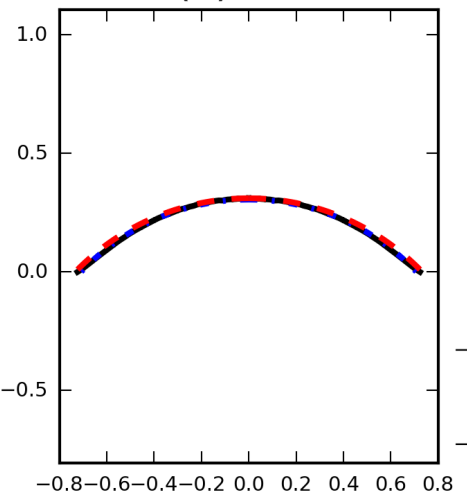

(c) $t=0.5$

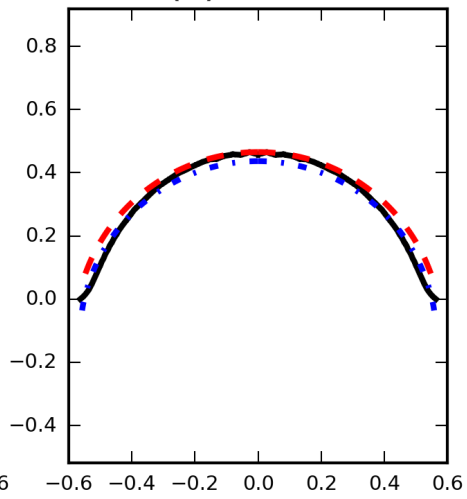

(f) $t=20$

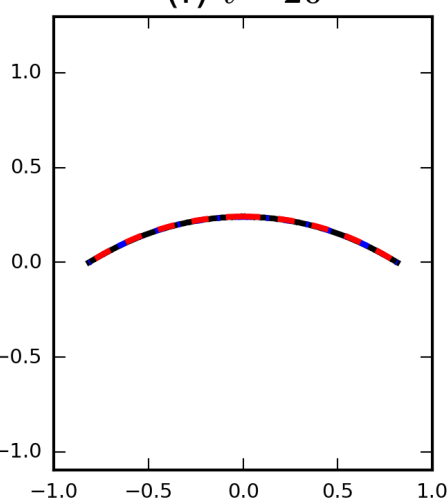

FIGURE 20 Drop shape during the spreading at different times equal to (a) $t=0$, (b) $t=0.1$, (c) $t=0.5$, (d) $t=2$, (e) $t=5$ and (f) $t=20$.

solutions, the apparent or dynamic contact angle $\theta_{d}$ is given by the following equation

$$
g\left(\theta_{d}, \hat{\eta}\right)-g\left(\theta_{s}, \hat{\eta}\right)=-\mathrm{Ca}^{*} \ln \epsilon,
$$

in which the function $g(\theta, \hat{\eta})$ is given by

$$
g(\theta, \hat{\eta})=\int_{0}^{\theta} \frac{d \alpha}{f(\alpha, \hat{\eta})}
$$

with

$$
f(\alpha, \hat{\eta})=\frac{2 \sin \alpha\left\{\hat{\eta}^{2}\left(\alpha^{2}-\sin ^{2} \alpha\right)+2 \hat{\eta}\left[\alpha(\pi-\alpha)+\sin ^{2} \alpha\right]+(\pi-\alpha)^{2}-\sin ^{2} \alpha\right\}}{\hat{\eta}\left(\alpha^{2}-\sin ^{2} \alpha\right)[\pi-\alpha+\sin \alpha \cos \alpha]+\left[(\pi-\alpha)^{2}-\sin ^{2} \alpha\right](\alpha-\sin \alpha \cos \alpha)} .
$$

The capillary number $\mathrm{Ca}^{*}$ is the normalized velocity of the moving contact line. Finally, the dimensionless parameter $\epsilon$ is the ratio to the slip length to the characteristic length which can be the drop diameter.

From our numerical solution, both the apparent (dynamic) contact angle and the capillary number $\mathrm{Ca}^{*}$ can be estimated. The dynamic contact angle is determined by the two solutions used to fit the drop shape in FIGURE 20. Since the velocity is normalized by the ratio $\sigma / \eta_{1}$, the dimensionless velocity close to the triple line can be used to estimated the capillary number $\mathrm{Ca}^{*}$. To see the behavior of the velocity field around the triple line, the velocity field is provided in FIGURE 21 for (a) $t=0.1$, (b) $t=0.5$, (c) $t=1$ and (d) $t=5$. At early stage, the velocity amplitude is important. While at $t=0.1$ the maximum of the velocity amplitude is around $1.5 \cdot 10^{-1}$ the magnitude of velocity decreases to $2.13 \cdot 10^{-2}$ when $t=5$. In the following, we estimate the capillary number $\mathrm{Ca}^{*}$ by taking the amplitude of the $r$-component of the velocity over the time range.

The dynamic contact angle has been determined using the approximate shapes described previously and depicted in FIGURE 22. For a large capillary number, the dynamic contact angle is larger than the static contact angle as expected by the theory. When the capillary number goes to zero, the dynamic contact angle becomes more and more close to the static contact 
(a) $t=0.1$

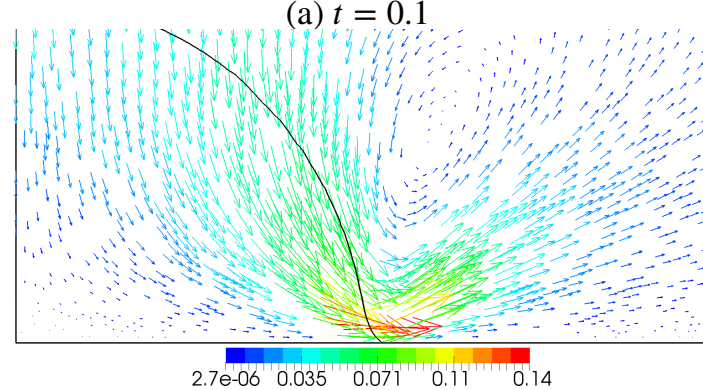

(c) $t=1$

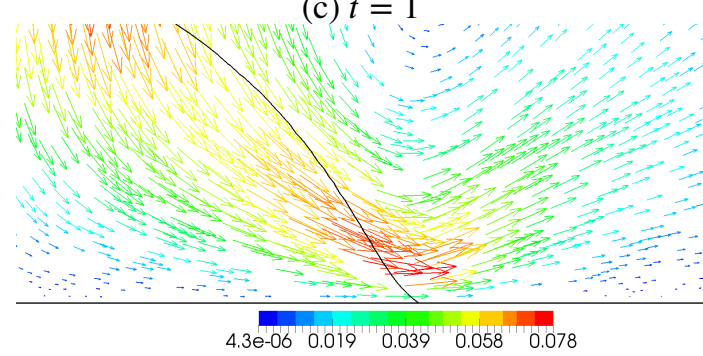

(b) $t=0.5$

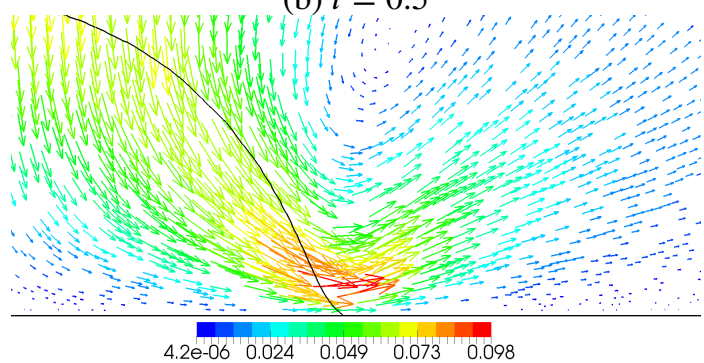

(d) $t=5$

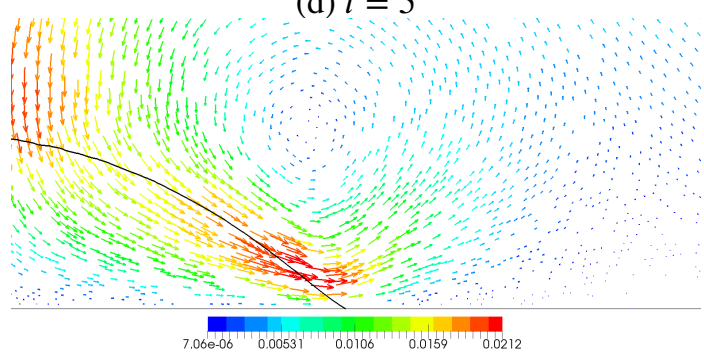

FIGURE 21 Velocity field in the neighbourhood of the triple line for a drop wetting a solid wall for $t=0.1,0.5,1$ and 5 .

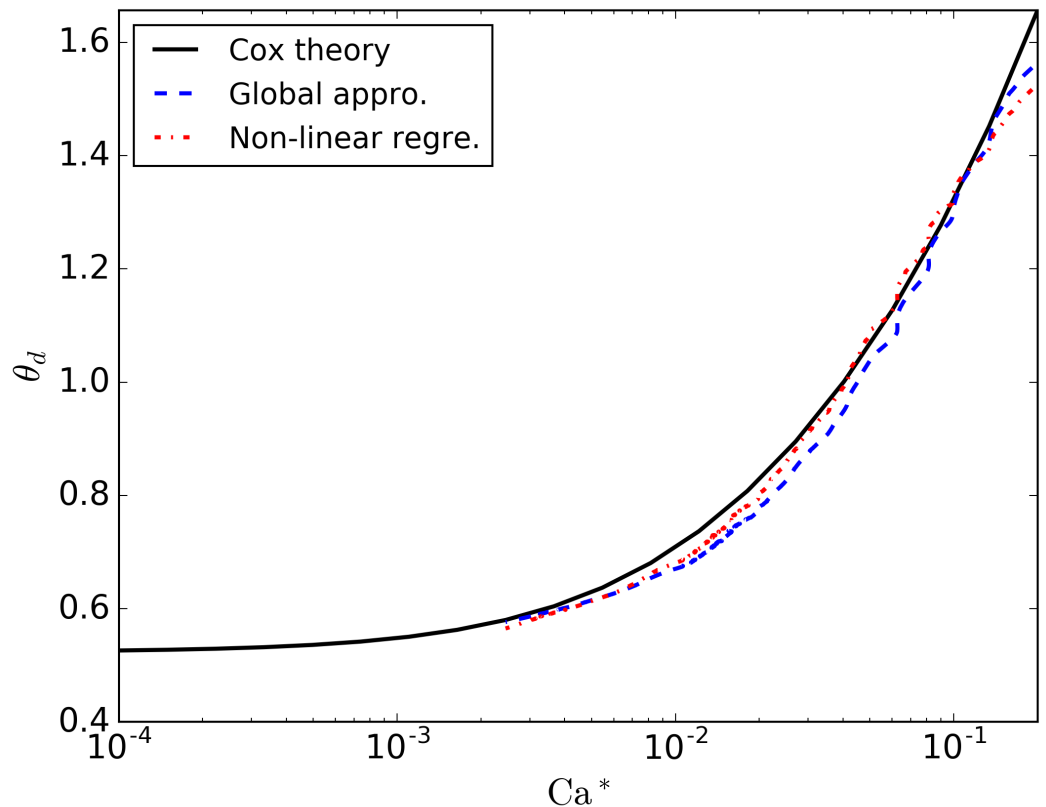

FIGURE 22 The dynamic contact angle as a function of $\mathrm{Ca}^{*}$ using the global approximation given in dashed line style and the non-linear regression given in dash-dot line style. The Cox theory solution is represented in solid line style for $\epsilon=10^{-1}$.

angle. The Cox's theory has been used to compare with our numerical solution. We adapt the value of the $\epsilon$ parameter set equal to $10^{-1}$ in order to have a good agreement. In first glance this value of $\epsilon$ seems very important in comparison with values classically admitted $\left(\sim 10^{-4}\right)$ according to Zahedi et al. ${ }^{69}$. Nevertheless, in our case, the slip length is not introduced in the numerical model. This "slip" length comes from the diffusion process of the chemical potential around the interface controlled by the value of the Péclet number as pointed out in the previous subsection. We showed that the diffusion characteristic length behaves as $1 / \sqrt[4]{\mathrm{Pe}}$ given an order of magnitude of $10^{-1}$ for a Péclet number equal to $10^{2}$ which is the order of magnitude used to set $\epsilon$. 
From this last case, the dynamics of wetting is clearly well captured with the Stokes/Cahn-Hilliard model even with a Cahn number equal to $10^{-2}$. The drop wetting on a solid substrate allows us to determine the dynamic contact angle with only one numerical run. The numerical results give the same trend that the Cox's theory.

\section{6 | CONCLUSION}

This work has been devoted to a development of a numerical method to solve the coupled Stokes/Cahn-Hilliard equations in the framework of discontinuous Galerkin finite element method. First, the numerical scheme of the Cahn-Hilliard equation has been developed. The coupled equations on the order parameter and on the chemical potential are solved using a method close to the one used to solve biharmonic equations. For a problem with a known exact solution, the spatial rate of convergence has been checked to be of optimal-order. Second, a numerical scheme for coupled Stokes/Cahn-Hilliard equations has been developed. This solver takes into account the dramatic variation of the viscosity. For a corresponding problem with known kinematic-phase field solution we also checked that the spatial and time schemes are both of optimal-order.

Three cases of two-phase flows have been investigated. First the droplet retraction in a liquid at rest has been numerically tested. Thanks to this test, we show that the Stokes/Cahn-Hilliard solver reproduces very well the drop retraction whatever the viscosity ratio used between the two liquids. Indeed, the comparison of the droplet retraction obtained numerically and with an approximate solution when droplets are slightly deformed is quite good. The droplet shape becomes more and more spherical exponentially with time. The retraction rate is a function of the viscosity ratio very well found with the numerical solver. In order to study the dynamics of the contact line physics, the problem of the capillary rising in a circular tube has been considered. The dynamics is very well reproduced and the asymptotic behavior of the static pressure equilibrium is captured. The scaling of the velocity close to the contact line has been studied. We pointed out that the diffusion scale of the chemical potential scales as a function of the fourth root of the Péclet number in agreement with the previous work of Briant and Yeomans ${ }^{65}$. Finally, the wetting dynamics is studied by investigating the spreading of a drop on a solid substrate. With only one computation, the dynamic contact angle can be determined as a function of the capillary number defined from the velocity of the contact line. The numerical results obtained in this work compare well with the theory established in the framework of the classical mechanics in which the triple line is a sharp-interface.

These results show that the discontinuous Galerkin formulation is adequate to solve the coupled Stokes/Cahn-Hilliard equations with a high level of accuracy. The interaction with walls is easily taken into account and provide predictions in agreement with the physics. The control of the diffusion of the chemical potential allows to mimic the slippage of the fluids close to the triple line. The characteristic length scale can be adapted by choosing the value of the Onsager mobility or the Péclet number. Nevertheless, to avoid a diffusion between fluid phases, the Cahn number must be sufficiently small and the domain must be limited in space in comparison with fluid inclusions investigated. Consequently, the Cahn-Hilliard theory is very well designed to study the fluid dynamics at a mesoscopic scale. In near future, this numerical solver will be used to study fluid interaction with heterogeneous walls and also generalize the formulation for non-Newtonian fluids.

How to cite this article: F. Pigeonneau, E. Hachem, and P. Saramito (2019), Discontinuous Galerkin finite element method applied to the coupled unsteady Stokes/Cahn-Hilliard equations, Int J Numer Meth Fluids, 2019;00:1-29.

\section{APPENDIX}

\section{A DETERMINATION OF THE SOURCE TERMS OF THE SPACE RATE OF CONVERGENCE}

\section{A.1 Source term on the Cahn-Hilliard equation}

The source term on the Cahn-Hilliard equation is obviously given by

$$
g(\boldsymbol{x}, t)=\frac{\partial \varphi}{\partial t}+\boldsymbol{\nabla} \varphi \cdot \boldsymbol{u}-\frac{1}{\mathrm{Pe}} \boldsymbol{\nabla} \mu .
$$


From the exact solution of $\varphi$, eq. (65) and those of $u$ and $v$, eqs. (67) and (68), the two components of the gradient of $\varphi$ are given by

$$
\begin{aligned}
& \frac{\partial \varphi}{\partial x}=-\pi u \\
& \frac{\partial \varphi}{\partial y}=\pi v .
\end{aligned}
$$

From these relations, the dot product $\boldsymbol{\nabla} \varphi \cdot \boldsymbol{u}$ is given by

$$
\boldsymbol{\nabla} \varphi \cdot \boldsymbol{u}=\pi \sin [\pi(x+y)] \sin [\pi(y-x)] \sin ^{2} t .
$$

The Laplacian of the chemical potential is easily determined from the exact solution of $\mu$ given by (66) which gives after the determination of the Laplacian of $\varphi^{3}$ the following relation

$$
\nabla^{2} \mu=-2 \pi^{2}\left(2 \pi^{2} \mathrm{Cn}^{2}-1\right) \varphi+6 \varphi \pi^{2} \sin ^{2} t\left[\sin ^{2}(\pi x) \cos ^{2}(\pi y)+\cos ^{2}(\pi x) \sin ^{2}(\pi y)-\cos ^{2}(\pi x) \cos ^{2}(\pi y)\right] .
$$

The source term $g(\boldsymbol{x}, t)$ is then easily determined by addition of the partial temporal derivative of $\varphi$ and the two equations (A4) and (A5).

\section{A.2 Source term of the Stokes equations}

The momentum equation taking into account the source term is written as follows

$$
-\boldsymbol{\nabla} p+\boldsymbol{\nabla} \cdot[2 \eta(\varphi) \boldsymbol{D}(\boldsymbol{u})]+\frac{3}{2 \sqrt{2} \mathrm{CaCn}} \mu \boldsymbol{\nabla} \varphi+\boldsymbol{f}=0 .
$$

As already pointed out in $\S 4.3$, the pressure gradient has not to be taken into account to determine the forced source term $\boldsymbol{f}$. So, the source term must take into account the viscous and the capillary forces. The equation solution of $u$ and $v$ gives that

$$
\begin{aligned}
\frac{\partial u}{\partial x} & =\pi \varphi, \\
\frac{\partial v}{\partial y} & =-\pi \varphi, \\
\frac{\partial u}{\partial y}=-\frac{\partial v}{\partial x} & =-\pi \sin (\pi x) \sin (\pi y) \sin t .
\end{aligned}
$$

From these relations, the rate-of-strain tensor is a diagonal tensor. So, the two components of $\boldsymbol{f}$ are given by

$$
\begin{gathered}
f_{x}=-2 \frac{\partial}{\partial x}\left[\eta(\varphi) \frac{\partial u}{\partial x}\right]-\frac{3}{2 \sqrt{2} \mathrm{CaCn}} \mu \frac{\partial \varphi}{\partial x}, \\
f_{y}=-2 \frac{\partial}{\partial y}\left[\eta(\varphi) \frac{\partial u}{\partial y}\right]-\frac{3}{2 \sqrt{2} \mathrm{CaCn}} \mu \frac{\partial \varphi}{\partial y} .
\end{gathered}
$$

Using the definition of the dynamic viscosity and the exact solutions of $\varphi$ and $u, f_{x}$ and $f_{y}$ take the following form

$$
\begin{gathered}
f_{x}=\pi^{2}[1+\hat{\eta}+2(1-\hat{\eta}) \varphi] u+\frac{3 \pi \mu u}{2 \sqrt{2} \mathrm{CaCn}}, \\
f_{y}=\pi^{2}[1+\hat{\eta}+2(1-\hat{\eta}) \varphi] v-\frac{3 \pi \mu v}{2 \sqrt{2} \mathrm{CaCn}},
\end{gathered}
$$

with $\varphi$ and $\mu$ given by (65) and (66).

\section{References}

1. Pozrikidis C.. Boundary integral and singularity methods for linearized viscous flow. Cambridge: Cambridge University Press; 1992.

2. Pigeonneau F., Sellier A.. Low-Reynolds-Number gravity-driven migration and deformation of bubbles near a free surface. Phys. Fluids. 2011;23:092102. 
3. Unverdi A. O., Tryggvason G.. A front-tracking method for viscous, incompressible, multi-fluid flows. J. Comput. Phys.. 1992;100:25-37.

4. Hirt C. W., Amsden A. A., Cook J. L.. An arbitrary Lagrangian-Eulerian computing method for all flow speeds. J. Comput. Phys.. 1974;14(3):227 - 253.

5. Girault V., López H., Maury B.. One time-step finite element discretization of the equation of motion of two-fluid flows. Numer. Methods Partial Differ. Equations. 2006;22(3):680-707.

6. Yue P., Feng J. J., Bertelo C. A., Hu H. H.. An arbitrary Lagrangian-Eulerian method for simulating bubble growth in polymer foaming. J. Comput. Phys.. 2007;226:2229-2249.

7. Ganesan S., Tobiska L.. A coupled arbitrary Lagrangian-Eulerian and Lagrangian method for computation of free surface flows with insoluble surfactants. J. Comput. Phys.. 2009;228(8):2859 - 2873.

8. Hirt C. W., Nichols B. D.. Volume of fluid (VOF) method for the dynamics of free boundaries. J. Comput. Phys.. 1981;39:201-226.

9. Sethian J. A.. Level set methods and fast marching methods. Evolving interfaces in computational geometry, fluid mechanics, computer vision, and materials science. Cambridge: Cambridge University Press; 1999.

10. Sethian J. A., Smereka P.. Level set methods for fluid interfaces. Annu. Rev. Fluid Mech.. 2003;35:341-371.

11. Legendre D., Maglio M.. Comparison between numerical models for the simulation of moving contact lines. Comput. Fluids. 2015;113:2-13.

12. Sui Y., Spelt P. D.M.. An efficient computational model for macroscale simulations of moving contact lines. J. Comput. Phys.. 2013;242:37-52.

13. Gross S., Reusken A.. Numerical methods for two-phase incompressible flows Springer series in computational mathematics, vol. 40: . Berlin: Springer-Verlag; 2011.

14. Enright D., Fedkiw R., Ferziger J., Mitchell I.. A Hybrid Particle Level Set Method for Improved Interface Capturing. J. Comput. Phys.. 2002;183(1):83 - 116.

15. Bui T. T. C., Frey P., Maury B.. A coupling strategy based on anisotropic mesh adaptation for solving two-fluid flows. Int. J. Numer. Methods Fluids. 2011;66(10):1226-1247.

16. Noble D. R., Newren E. P., Lechman J. B.. A conformal decomposition finite element method for modeling stationary fluid interface problems. Int. J. Numer. Methods Fluids. 2010;63(6):725-742.

17. Fries T.-P.. Higher-order conformal decomposition FEM (CDFEM). Comput. Methods Appl. Mech. Eng.. 2018;328:75-98.

18. Huh C., Scriven L. E.. Hydrodynamic model of steady movement of a solid/liquid/fluid contact line. J. Colloid Interface Sci.. 1971;35(1):85-101.

19. Cox R. G.. The dynamics of the spreading of liquids on a solid surface. Part 1. Viscous flow. J. Fluid Mech.. 1986;168:169194.

20. Cahn J. W., Hilliard J. E.. Free Energy of a Nonuniform System. I. Interfacial Free Energy. J. Chem. Phys.. 1958;28(2):258267.

21. Anderson D. M., McFadden G. B., Wheeler A. A.. Diffuse-interface methods in fluid Mechanics. Annu. Rev. Fluid Mech.. 1998;30:139-165.

22. Antanovskii L. K.. A Phase Field Model of Capillarity. Phys. Fluids. 1995;7(4):747-753.

23. Yue P., Feng J. J., Liu C., Shen J.. Diffuse-interface simulations of drop coalescence and retraction in viscoelastic fluids. $J$. Non-Newtonian Fluid Mech.. 2005;129(3):163-176. 
24. Lowengrub J., Truskinovsky L.. Quasi-incompressible Cahn-Hilliard fluids and topological transitions. Proc. R. Soc. Lond. A. 1998;454:2617-2654.

25. Mauri R., ed.Multiphase microfluidics: the diffuse interface model CISM Courses and Lectures, vol. 538: . Udine: Springer; 2012.

26. Seppecher P.. Moving contact lines in the Cahn-Hilliard theory. Int. J. Engng Sci.. 1996;34(9):977-992.

27. Jacqmin D.. Contact-line dynamics of a diffuse fluid interface. J. Fluid Mech.. 2000;402:57-88.

28. Qian T., Wang X.-P., Sheng P.. A variational approach to moving contact line hydrodynamics. J. Fluid Mech.. 2006;564:333.

29. Villanueva W., Amberg G.. Some generic capillary-driven flows. Int. J. Multiphase Flow. 2006;32:1072-1086.

30. Biben T., Joly L.. Wetting on nanorough surfaces. Phys. Rev. Lett.. 2008;100(18):186103.

31. Yue P., Zhou C., Feng J. J.. Sharp-interface limit of the Cahn-Hilliard model for moving contact lines. J. Fluid Mech.. 2010;645:279-294.

32. Yue P., Feng J. J.. Wall energy relaxation in the Cahn-Hilliard model for moving contact lines. Phys. Fluids. 2011;23(1):012106.

33. Jacqmin D.. Calculation of Two-Phase Navier-Stokes Flows Using Phase-Field Modeling. J. Comput. Phys.. 1999;155(1):96-127.

34. Badalassi V. E., Ceniceros H. D., Banerjee S.. Computation of multiphase systems with phase field models. J. Comput. Phys.. 2003;190:371-397.

35. Ta M., Pigeonneau F., Saramito P.. An implicit high order discontinuous Galerkin level set method for two-phase flow problems. 9th International Conference on Multiphase Flow:1-6; 2016; Firenze, Italy.

36. Di Pietro D. A., Ern A.. Mathematical aspects of discontinuous Galerkin methods. Heidelberg: Springer-Verlag; 2012.

37. Badia S., Codina R., Gudi T., Guzmán J.. Error analysis of discontinuous Galerkin methods for the Stokes problem under minimal regularity. IMA J. Numer. Anal.. 2014;34(2):800-819.

38. Feng X., Karakashian O. A.. Fully discrete dynamic mesh discontinuous Galerkin methods for the Cahn-Hilliard equation of phase transition. Math. Comp.. 2007;76:1093-1117.

39. Baker G. A.. Finite element methods for elliptic equations using nonconforming elements. Math. Comput.. 1977;31(137):45-59.

40. Kay D., Styles V., Süli E.. Discontinuous Galerkin finite element approximation of the Cahn-Hilliard equation with convection. SIAM J. Numer. Anal.. 2009;47(4):2660-2685.

41. Gudi T., Nataraj N., Pani A. K.. Mixed Discontinuous Galerkin Finite Element Method for the Biharmonic Equation. J. Sci. Comput.. 2008;37:139-161.

42. Wells G. N., Kuhl E., Garikipati K.. A discontinuous Galerkin method for the Cahn-Hilliard equation. J. Comput. Phys.. 2006;218(2):860-877.

43. Liu C., Rivière B. Numerical analysis of a discontinuous Galerkin method for Cahn-Hilliard-Navier-Stokes equations. arXiv e-prints. 2018;:arXiv:1807.02725.

44. Cahn J. W.. Critical point wetting. J. Chem. Phys.. 1977;66(8):3667-3672.

45. Waals J. D.. The thermodynamic theory of capillarity under the hypothesis of a continuous variation of density. Verhandel. Konink. Akad. Weten.. 1893;1:1-56.

46. De Gennes P.-G., Brochard-Wyart F., Quéré D.. Gouttes, bulles, perles et ondes. Paris: Belin; 2005. 
47. Giaquinta M., Hildebrandt S.. Calculus of variations I. Berlin: Springer-Verlag; 1996.

48. Hohenberg P. C., Halperin B. I.. Theory of dynamic critical phenomena. Rev. Mod. Phys.. 1977;49(3):435-479.

49. Gurtin M. E., Polignone D., Vinals J.. Two-phase binary fluids and immiscible fluids described by an order parameter. Math. Models Meth. Appl. Sci.. 1996;6(6):815-831.

50. Matsumoto S., Maruyama S., Saruwatari H.. A molecular dynamics simulation of a liquid droplet on a solid surface. . Proc. ASME-JSME Thermal Engng Joint Conf. 1995:557-562.

51. Arnold D.. An Interior Penalty Finite Element Method with Discontinuous Elements. SIAM J. Numer. Anal.. 1982;19(4):742-760.

52. Castillo P.. Performance of discontinuous Galerkin methods for elliptic PDEs. SIAM J. Sci. Comput.. 2002;24(2):524-547.

53. Shahbazi K.. An explicit expression for the penalty parameter of the interior penalty method. J. Comput. Phys.. 2005;205(2):401-407.

54. Süli E., Mayers D. F.. An Introduction to Numerical Analysis. Cambridge (UK): Cambridge University Press; 2003.

55. Saramito Pierre. Efficient C++ finite element computing with Rheolef. CNRS-CCSD ed.; version 7.0 ed.2018.

56. Yue P., Zhou C., Feng J. J.. Spontaneous shrinkage of drops and mass conservation in phase-field simulations. J. Comput. Phys.. 2007;223(1):1-9.

57. Cockburn B., Kanschat G., Schötzau D., Schwab C.. Local discontinuous Galerkin methods for the Stokes system. SIAM J. Numer. Anal.. 2002;40(1):319-342.

58. Abramowitz M., Stegun I. A.. Handbook of mathematical functions. New York: Dover Publications, Inc.; 1965.

59. Dong S., Shen J.. A time-stepping scheme involving constant coefficient matrices for phase-field simulations of two-phase incompressible flows with large density ratios. J. Comput. Phys.. 2012;231(17):5788-5804.

60. Castro-Díaz M. J., Hecht F., Mohammadi B., Pironneau O.. Anisotropic unstructured mesh adaption for flow simulations. Int. J. Num. Meth. Fluids. 1997;25(4):475-491.

61. Hecht F.. BAMG: bidimensional anisotropic mesh generator. User Guide: INRIAParis; 2006.

62. Oldroyd J. G.. On the formulation of rheological equations of state. Proc. R. Soc. Lond. A. 1950;200(1063):523-541.

63. Frankel N. A., Acrivos A.. The constitutive equation for a dilute emulsion. J. Fluid Mech.. 1970;44(01):65-78.

64. Maffetone P. L., Minale M.. Equation of change for ellipsoidal drops in viscous flow. J. Non-Newtonian Fluid Mech.. 1998; 78:227-241.

65. Briant A. J., Yeomans J. M.. Lattice Boltzmann simulations of contact line motion. II. Binary fluids. Phys. Rev. E. 2004;69:031603.

66. Van Dyke M.. Perturbations methods in fluid mechanics. Stanford, California: The parabolic Press; 1975.

67. De Gennes P. G.. Wetting: Statics and dynamics. Rev. Mod. Phys.. 1985;57(3):827-863.

68. Hoffman R. L.. A study of the advancing interface. I. Interface shape in liquid-gas systems. J. Colloid Interface Sci.. 1975;50(2):228-241.

69. Zahedi S., Gustavsson K., Kreiss G.. A conservative level set method for contact line dynamics. J. Comput. Phys.. 2009;228(17):6361 - 6375. 